\title{
Edem1 activity in the fat body regulates insulin signalling and metabolic homeostasis in Drosophila
}

\author{
Himani Pathak (1), Jishy Varghese (1)
}

\begin{abstract}
In Drosophila, nutrient status is sensed by the fat body, a functional homolog of mammalian liver and white adipocytes. The fat body conveys nutrient information to insulin-producing cells through humoral factors which regulate Drosophila insulinlike peptide levels and insulin signalling. Insulin signalling has pleiotropic functions, which include the management of growth and metabolic pathways. Here, we report that Edem1 (endoplasmic reticulum degradation-enhancing $\alpha$-mannosidase-like protein 1), an endoplasmic reticulum-resident protein involved in protein quality control, acts in the fat body to regulate insulin signalling and thereby the metabolic status in Drosophila. Edem1 limits the fat body-derived Drosophila tumor necrosis factor- $\alpha$ Eiger activity on insulin-producing cells and maintains systemic insulin signalling in fed conditions. During food deprivation, edem1 gene expression levels drop, which aids in the reduction of systemic insulin signalling crucial for survival. Overall, we demonstrate that Edem1 plays a vital role in helping the organism to endure a fluctuating nutrient environment by managing insulin signalling and metabolic homeostasis.
\end{abstract}

DOI 10.26508/Isa.202101079 | Received 29 March 2021 | Revised 3 June 2021 | Accepted 7 June 2021 | Published online 17 June 2021

\section{Introduction}

Energy homeostasis, the sum of all processes which maintain the balance between energy inflow and outflow; is vital for normal functioning, reproduction as well as longevity. Energy homeostasis in animals is brought about by the activity and interplay of various endocrine and neuroendocrine systems. Insulin/insulin-like growth factor (IGF) signalling pathway plays a significant role in the maintenance of energy balance and is well conserved in both vertebrates and invertebrates (Kenyon et al, 1993; Kimura et al, 1997; Brogiolo et al, 2001; Clancy et al, 2001; Fabrizio et al, 2001; Britton et al, 2002; Fernandez \& Torres-Aleman, 2012). The perturbations in insulin signalling result in a plethora of effects, such as; diabetes (Britton et al, 2002; Rulifson et al, 2002), obesity (Kahn et al, 2006), reduced body size (Liu et al, 1993; Accili et al, 1996; Ikeya et al, 2002; Rulifson et al, 2002), resistance to starvation and oxidative stress, and life span effects (Clancy et al, 2001; Tatar et al, 2001; Bonafè et al, 2003; Holzenberger et al, 2003; Shimokawa et al, 2003; Giannakou et al, 2004; Katic \& Kahn, 2005; Sonntag et al, 2005; Giannakou \& Partridge, 2007). Drosophila melanogaster, a widely used genetic model organism, has eight insulin-like peptides (Drosophila insulin-like peptide [DILPS] 1-8), which share structural and functional similarities with mammalian insulin and IGFs (Grönke et al, 2010). Among these DILPS; DILP2, DILP3, and DILP5 are produced mainly by a subset of the median neurosecretory cells (mNSCs), the insulin-producing cells (IPCS), in the fly brain (Ikeya et al, 2002; Géminard et al, 2009; Broughton et al, 2010; Nässel, 2012). The major effector tissue of insulin signalling is the fat body, which is also the main energy reserve and nutrient sensor in flies (Hwangbo et al, 2005; Géminard et al, 2009). The fat body relays information about the nutrient status of the organism through humoral factors, which act on the IPCS directly or indirectly to control systemic insulin signalling (Colombani et al, 2003; Géminard et al, 2009; Droujinine \& Perrimon, 2016). The fat-derived signals that control IPC function include DILP6 (Bai et al, 2012), a DILP; unpaired2 (Upd2), a functional homolog of leptin in Drosophila and activator of JAK-STAT pathway (Rajan \& Perrimon, 2013); Eiger, the Drosophila tumor Necrosis Factor $\alpha /$ TNF $\alpha$, which activates JNK signalling (Agrawal et al, 2016); CCHamide2 (Sano et al, 2015), a nutrient responsive peptide hormone; growth-blocking peptide (GBP) (Koyama \& Mirth, 2016), a Drosophila cytokine; Stunted, a circulating insulinotropic peptide (Delanoue et al, 2016); female-specific independent of transformer (FIT) (Sun et al, 2017); and activin-like ligand Dawdle (Ghosh \& O'Connor, 2014). The molecular mechanisms that regulate the synthesis and secretion of the fat body-derived signals (FDSs) are currently under intense investigation.

The ER serves many functions in the eukaryotic cell, foremost of which is the synthesis and folding of nascent proteins with the help of molecular chaperones and folding enzymes. Hence, the ER is considered as the major quality-control site which ensures that only correctly folded proteins are allowed to leave to other cellular compartments. The ER is also considered to be the first storage site of secretory proteins and the ER activity is high in cells of endocrine and exocrine tissues because of the heavy protein trafficking in such cells. Genetic factors, physiological changes, and fluctuations in the cellular environment might lead to misfolding of proteins (Liu

School of Biology, Indian Institute of Science Education and Research (IISER TVM) Thiruvananthapuram, Kerala, India

Correspondence: jishy@iisertvm.ac.in 
\& Kaufman, 2003) and the ER aids in eliminating proteins, which remain misfolded even after multiple rounds of folding attempts. Thus, a proper balance between the influx of proteins and the folding machinery in the ER is crucial for efficient protein quality control. When the ER homeostasis is upset misfolded proteins accumulate in the ER triggering an adaptive response called unfolded protein responses (UPR). The UPR signalling mainly involves three ER residing transmembrane sensors: inositolrequiring protein 1 , activating transcription factor 6 , and PKR-like ER kinase (PERK). The UPR sensors would initiate ER-associated degradation (ERAD) of terminally misfolded proteins, expand the ER membrane, increase the folding capacity of the ER, and decrease the overall protein load in the ER (Liu \& Kaufman, 2003). Permanently unfolded glycoproteins are recognised by ERAD-enhancing $\alpha$-mannosidase-like proteins (Edem), which aid in the degradation of the misfolded proteins (Molinari et al, 2003; Araki \& Nagata, 2011; Kroeger et al, 2012). Glycoproteins constitute a large proportion of proteins in a cell; hence, the function of Edem is crucial for cellular homeostasis.

Here, we report that Edem1 activity in the Drosophila fat body is crucial for maintaining systemic insulin signalling. Down-regulation of edem 1 gene expression in the fat body resulted in the accumulation of DILP2 in the IPCs, a decrease in dilp3 mRNA levels and reduced systemic insulin signalling, which led to nutrient imbalances and altered sensitivity to starvation. Our results also show that Edem1 regulates fat body-derived Drosophila TNFa Eiger activity on the IPCs, crucial for managing systemic insulin signalling and metabolic status. Activation of target of rapamycin (TOR) signalling, the main amino acid sensor, and a key regulator of Eiger activity rescued the effects of edem 1 down-regulation. In addition, we report that Edem1 activity in the fat body regulate Upd2, another fat body-derived cytokine, to manage metabolic status. Furthermore, in response to nutrient deprivation, edem1 transcripts were found to be low, which we show is critical to the reduction in systemic insulin levels and better survival of flies during starvation. We propose that Edem 1 acts as a key factor in the fat body, which maintains nutrient homeostasis by controlling the activity of the IPCS through Eiger.

\section{Results}

\section{Edem1 maintains metabolic homeostasis}

We embarked on a large-scale genetic screen in Drosophila to identify factors that control nutrient homeostasis and insulin signalling. Towards this, we blocked various candidate genes, reported to be differentially expressed in the miR-14 mutants that exhibited metabolic imbalances, in the Drosophila fat body using RNAi lines (Varghese et al, 2010). We chose male flies for this study to minimize the effects of oogenesis on nutrient homeostasis. In this screen, we identified Edem1, an ER-resident protein involved in protein quality control, as a putative regulator of metabolic status in Drosophila. Down-regulation of edem 1 transcripts in the fat body led to a significant increase in the levels of energy storestriglycerides and glycogen-in 5-d-old adult flies (Fig 1A and B). In response to knock down of edem1 in the fat body, flies survived longer in response to acute nutrient deprivation (Fig 1C). We chose 5 -d-old flies to completely avoid the influence of larval fat cells which persists in adult flies for few days after eclosion. We have confirmed the effects of blocking Edem1 in the fat body using independent RNAi lines, which rules out off-target effects and insertional site-specific effects (Fig S1B and C). We have also downregulated edem 1 expression with an independent fat body driver CgGal4 to make sure that the effect is coming because of fat body-specific knock down of edem1 (Fig S4A-H). We could replicate most of the experiments from Fig 1 with the CgGal4 driver as well. The higher energy stores present in response to reduction in edem 1 levels in the fat body, and excess energy stores mobilized could account for the better survival of flies during nutrient deprivation (Fig 1D and E). Along with changes in stored nutrient levels in adult flies, circulating glucose levels were high in the larval hemolymph (Fig 1F). In addition, blocking edem1 in the fat body led to enhanced feeding responses in the larvae (Fig 1G), similar to responses reported earlier in food deprived larvae and also in response to low insulin because of its anorexigenic effects (Zhang et al, 2013; Chouhan et al, 2017). We also observed an increase in life span of the adult flies upon edem1 down-regulation in the fat body (Fig 1H). These data show that Edem1 function in the fat body is crucial in regulating metabolic homeostasis in Drosophila. The phenotypes observed in response to blocking edem1 levels on larval circulating sugar levels, larval feeding, adult energy stores, and life span indicated a reduction in insulin signalling, as reported by earlier studies. Reduction in the levels of DILPS produced by IPCs led to the accumulation of triglycerides and glycogen (Grönke et al, 2010; Bai et al, 2012). Ablation of IPCs also resulted in higher levels of circulating sugars, glycogen, lipid stores, and enhanced resistance to food deprivation (Rulifson et al, 2002; Broughton et al, 2005; Haselton et al, 2010). In addition, there is proof that defects in insulin signalling led to reduced adult body size and excess fat storage (Böhni et al, 1999; Tatar et al, 2001; Shingleton et al, 2005; Slaidina et al, 2009). miR-278 mutants have elevated dilp2, 3, and 5 transcript levels and are lean, whereas miR-14 mutants are obese, because of reduced dilp transcript levels and insulin signalling (Teleman et al, 2006; Varghese et al, 2010). In addition, reduced insulin signalling is crucial for starvation triggered foraging and activation of IPCS or overexpression of DILPs led to less food intake (Wu et al, 2005; Hong et al, 2012). Hyperactivation of insulin signalling induced satiation in larvae (Britton et al, 2002). Also, reduced insulin signalling (Clancy et al, 2001; Tatar et al, 2001; Bai et al, 2012) and ablation of IPCS, extended life span (Broughton et al, 2005; Haselton et al, 2010). However, we did not observe any developmental growth effects as expected in response to reduced insulin signalling. Next, we tested if insulin signalling is reduced in response to blocking edem 1 levels in the fat body.

\section{Edem1 function in the fat body maintains systemic insulin signalling}

To measure the insulin signalling activity in response to blocking edem 1 in the fat body, we checked gene expression of key downstream target genes of insulin pathway. Transcription of $4 e b p$ (elF4E-binding protein), inr (insulin receptor), and dilp6 is suppressed by insulin signalling and these insulin target genes can be 


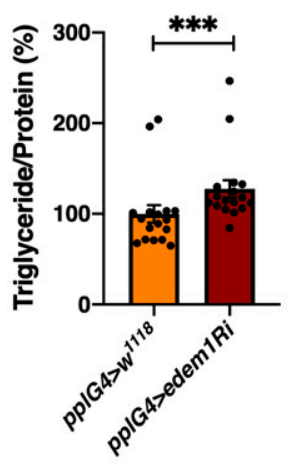

E

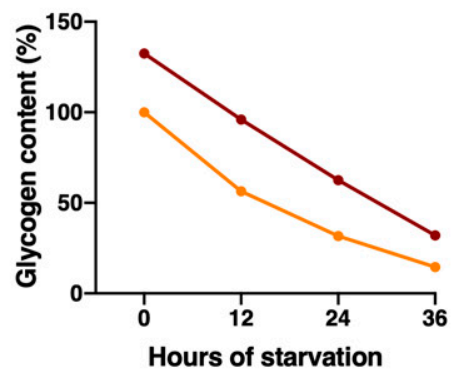

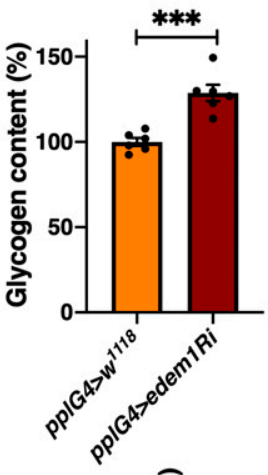

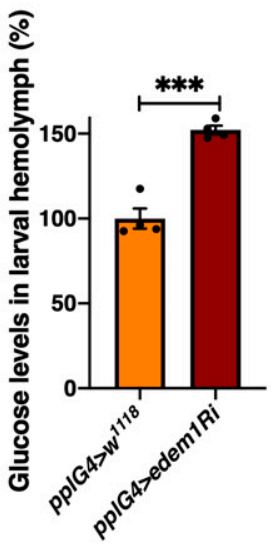

$\rightarrow p p / G 4>w^{1118}$

$\rightarrow$ pp/G4>edem1Ri

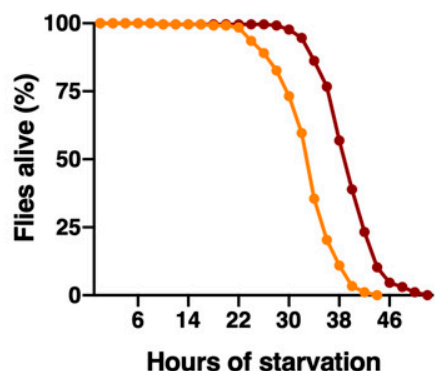

G

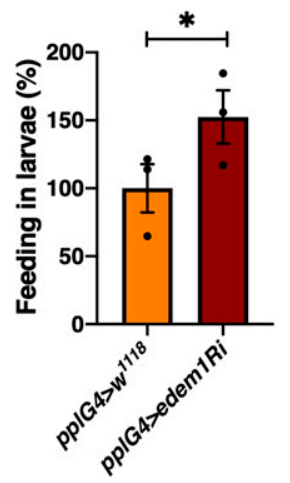

D

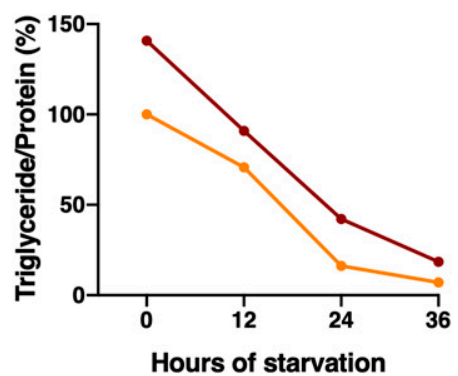

H

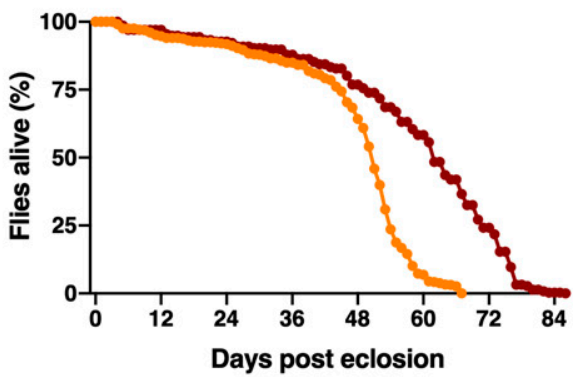

Figure 1. Edem1 maintains metabolic homeostasis.

(A) Blocking edem1 expression using RNAi in the fat body led to enhanced triglyceride levels in adult 5 -d-old male flies. Data are shown as \% ratio of triglyceride to total protein levels, normalised to $100 \%$ in pplGal $4>W^{1118}$ (control) and increase in experimental conditions pplGal $4>U A S$-edem1-RNAi (independent biological replicates $=17$, $P$-value between control and UAS-edem1-RNAi is $<0.001$ [Mann-Whitney test]). (B) Enhanced levels of glycogen in adult 5-d-old male flies caused by blocking edem1 expression in the fat body. Data are shown as \% of total glycogen levels, normalised to $100 \%$ in pplGal $>>w^{1118}$ (control), and increase in experimental conditions pplGal4>UAS-edem1-RNAi (independent biological replicates $=6, P$-value between control and UAS-edem1-RNAi is $<0.001$ [Mann-Whitney test]). (C) Enhanced resistance to starvation in adult 5-d-old male flies caused by blocking edem1 expression in the fat body. Data shown as percentage of flies of pplGal4>w1118 (control) and pplGal4>UAS-edem1-RNAi which were alive at various time points of starvation (independent biological replicates $=4$, number of flies used for control is 255 and for pplGal4>UAS-edem1-RNAi is 262. P-value between control and UAS-edem1-RNAi is $<0.001$ [log-rank test], Wald test $=189.8$ on $\mathrm{df}=1, P<0.001$ [Cox hazard proportional analysis]). (D, E) Utilisation of triglycerides and glycogen at different stages of starvation upon edem 1 knock down. Data are shown as \% ratio of triglyceride to total protein levels in adult male flies, data are normalised to $100 \%$ in pplGal $>>W^{1118}$ (control) fed condition and change in response to indicated hours of starvation in control and experimental conditions pplGal4>UAS-edem1-RNAi is shown (independent biological replicates $=3, P$-value between control and UAS-edem1-RNAi is 0.3844 [log-rank test]). Glycogen levels at different stages of starvation upon edem1 knock down. Data are normalised to $100 \%$ in pplGal $>>W^{1118}$ (control) fed condition and change in response to indicated hours of starvation in control and experimental conditions pplGal $>>$ UAS-edem1-RNAi is shown (independent biological replicates $=3$, $P$-value between control and UAS-edem1-RNAi is 0.0082 [log-rank test]). (F) Expression of edem1-RNAi in the fat body led to enhanced glucose levels in the circulation. Data are shown as \% of glucose levels in the hemolymph, normalised to $100 \%$ in pplGal $4>W^{1118}$ (control) and increase in experimental conditions pplGal4>UAS-edem1-RNAi (independent biological replicates $=4, P$-value between control and UAS-edem1-RNAi is $<0.001$ [Mann-Whitney test]). (G) Blocking edem1 gene expression in the fat body led to enhanced feeding responses in larvae. Data are shown as \% food consumption in larvae, normalised to $100 \%$ in pplGal $4>w^{1118}$ (control) and increase in experimental conditions pplGal4>UAS-edem1-RNAi (independent biological replicates $=3$, P-value between control and UAS-edem1-RNAi is 0.0329 [Welch's $t$ test]). (H) edem1-RNAi in the fat body led to enhanced life span in adult male flies. Data are shown as percentage of input flies pplGal4> $w^{1118}$, pplGal4>UAS-edem1-RNAi which were alive across the days (independent biological replicates $=3$, number of flies used for control is 453 and for pplGal $4>U A S$-edem1-RNAi is 372 . P-value between control and UAS-edem1-RNAi is $<0.001$ [log-rank test], Wald test $=275.1$ on df $=1, P<0.001$ [Cox hazard proportional analysis]). (A, B, F, G) $\left(P\right.$-value ${ }^{*}<0.05 ;{ }^{* *}<0.01$, ${ }^{* * *}<0.001 ;$ Data information: In $[A, B, F, G]$ data are presented as mean $\pm S E M)$.

Source data are available for this figure.

used as a read out for insulin signalling activity (Puig et al, 2003; Slaidina et al, 2009). Blocking edem1 in the fat body increased transcript levels of the insulin responsive genes, which indicate low insulin signalling (Fig 2A). We speculated if Edem1 activity in the fat body could regulate IPC function and control systemic insulin signalling, as fat body is known to remotely control IPCs. To address whether Edem 1 in the fat body regulates IPC function, the transcript levels of IPC specific DILPs-dilp2, dilp3, and dilp5 were measured in the late third instar larval stage. In response to the expression of edem1-RNAi in the fat body, dilp3 mRNA levels were found to be low; however, there were no detectable changes in the mRNA levels of dilp2 and dilp5 (Fig 2B). Previous studies report that nutrient deprivation would block DILP secretion from the IPCS into the hemolymph leading to an accumulation of DILPs and reduction in systemic insulin signalling (Géminard et al, 2009). We observed an increase in DILP2 puncta in IPCS in response to reducing edem1 

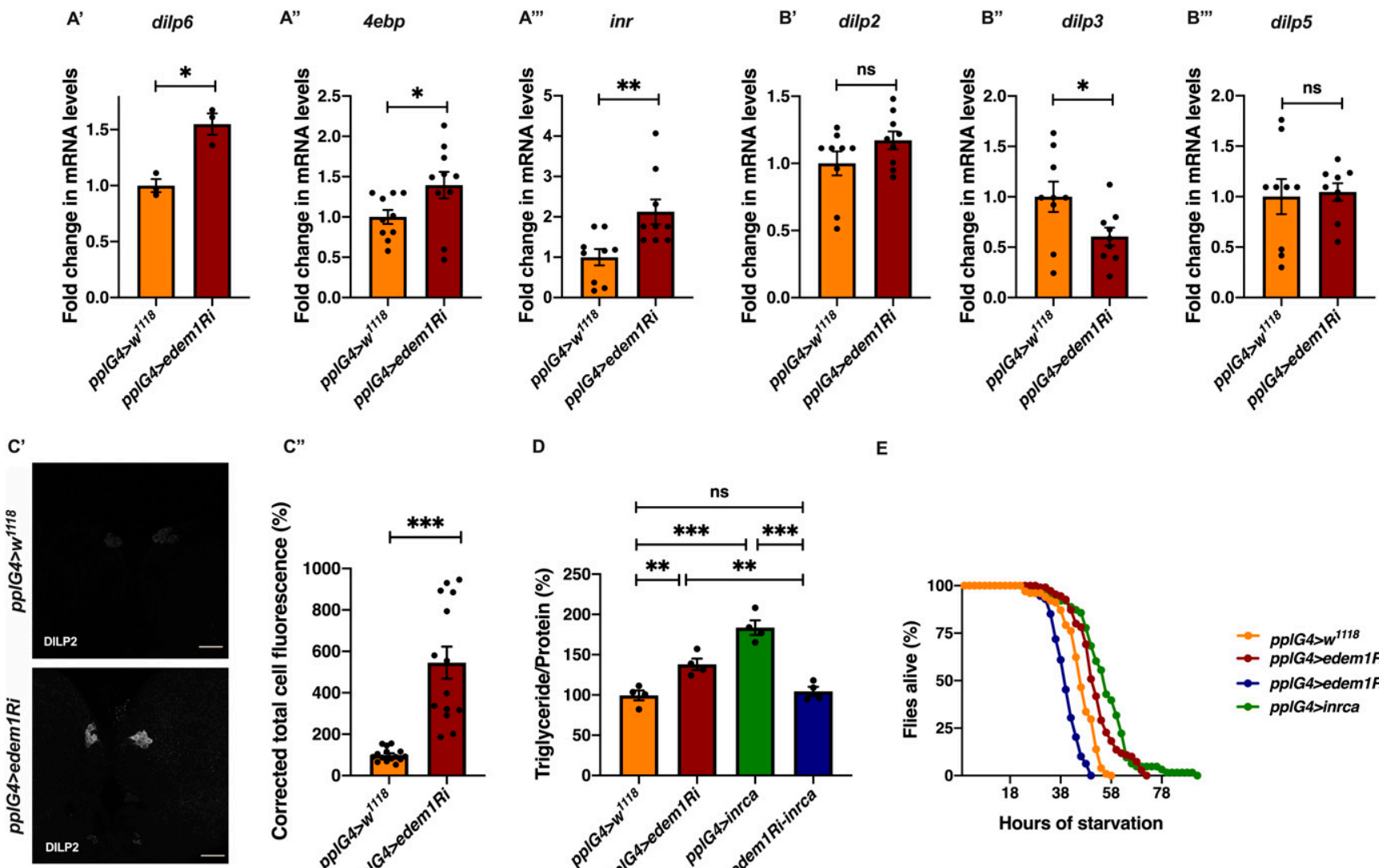

C"

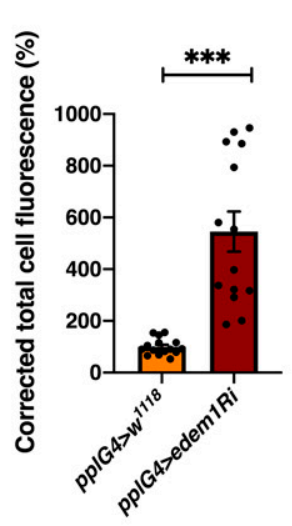

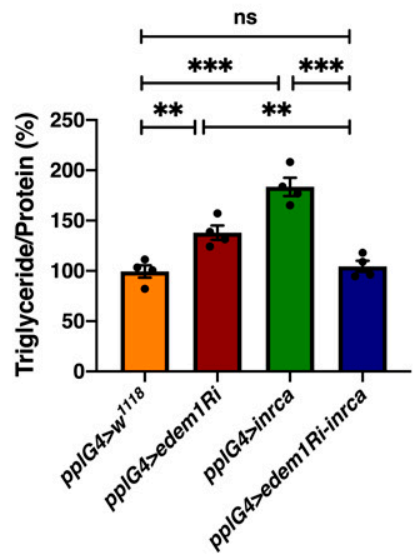

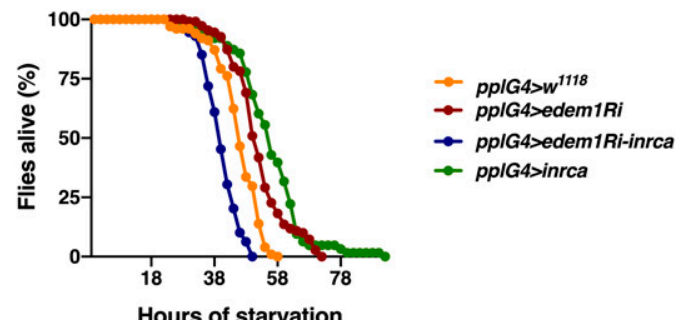

Figure 2. Blocking edem1 in the fat body reduced insulin signalling.

(A) Blocking edem1 expression using RNAi in the fat body led to an increase in mRNA levels of insulin target genes dilp6 ( $\left.A^{\prime}\right)$, 4 ebp ( $\left.A^{\prime \prime}\right)$ and inr ( $\left.A^{\prime \prime \prime}\right)$ in larvae. Data are shown as fold change in mRNA levels, values are normalised to pplGal $>>W^{1118}$, and fold change in pplGal $4>U A S-$-edem1-RNAi is shown (independent biological replicates = $3, P$-value between control and UAS-edem1-RNAi is 0.0128 for dilp6 [Welch's $t$ test], for 4 ebp independent biological replicates $=10$ and $P$-value is 0.0473 [unpaired $t$ test], for inr independent biological replicates $=9$ and $P$-value is 0.0087 [Welch's $t$ test]). (B) Blocking edem1 expression using RNAi in the fat body also led to a decrease in the levels of insulin-producing cell specific dilp3 mRNA in larvae. Data are shown as fold change in mRNA levels, values are normalised to pplGal $4>w^{1118}$, and fold change in pplGal4>UAS-edem1-RNAi is shown. ( $n=9, P$-value between control and UAS-edem1-RNAi is 0.1809 for dilp2 [Mann-Whitney test], 0.0432 for dilp3 [Welch's t test] and 0.8187 for dilp5 [Welch's $t$ test]). (C) DILP2 protein in the larval insulin-producing cells shown as a representative image (C') of anti-DILP2 antibody staining in larval brains of pplGal4> $W^{1118}$ (independent biological replicates $=15$ ) and pplGal4>UAS-edem1-RNAi (independent biological replicates $=14$ ). Corrected total cell fluorescence values are normalised to pplGal4>W ${ }^{1118}$, and fold change in pplGal4>UAS-edem1-RNAi is shown in ( $\left.C^{\prime \prime}\right)$ ( $P$-value between control and UAS-edem1-RNAi is <0.001 [Mann-Whitney test]) (Scales: $30 \mu \mathrm{m}$ ). (D) Overexpression of a constitutively active form of inr (InR ${ }^{A 1325 D}$ ) with edem1-RNAi in the fat body led to the rescue of fat phenotype in adult male flies. Data is shown as \% ratio of triglyceride to total protein levels, normalised to $100 \%$ in pplGal $>W^{1118}$ (control) and changes in experimental conditions pplGal $>$ UAS-edem 1 RNAi, pplGal4>UAS-InR ${ }^{A 1325 D}$ and pplGal4>UAS-edem1-RNAi; UAS-InR ${ }^{A 1325 D}$ (independent biological replicates $=4, P$-value between control and UAS-edem1-RNAi is $<0.001$, $P$-value between control and UAS-InR $R^{A 1325 D}$ is $<0.001$, P-value between UAS-edem1-RNAi and UAS-edem1-RNAi, UAS-InR $R^{A 1325 D}$ is $0.001, P$-value between UAS-edem1-RNAi and UAS-InR $R^{A 1325 D}$ is 0.1292, P-value between UAS-InR ${ }^{A 1325 D}$ and UAS-edem1-RNAi, UAS-InR $R^{A 1325 D}$ is $<0.001$ and $P$-value between control and UAS-edem1-RNAi, UAS-InR $R^{A 1325 D}$ is $>0.9999$ [Kruskal-Wallis test followed by Dunn's post hoc test]). (E) Starvation resistance in adult 5-d-old male flies shown as percentage of input flies pplGal4>W1118, pplGal4>UAS-edem1-RNAi, pplGal4>UAS-InR $R^{A 1325 D}$ and pplGal4> UAS-edem1-RNAi, UAS-InR ${ }^{A 1325 D}$ which were alive at various time points of starvation (independent biological replicates $=3$, number of flies used for control is 77, for pplGal4>UAS-edem1-RNAi is 110, for pplGal4>UAS-InR ${ }^{A 1325 D}$ is 126 and for $p$ plGal4> UAS-edem1-RNAi, UASInR $R^{A 1325 D}$ is 128. P-value between control and UAS-edem1-RNAi is $<0.001$, P-value between control and UAS-InR ${ }^{A 1325 D}$ is $\angle 0.001, P$-value between UAS-edem1-RNAi and UASedem1-RNAi, UAS-InR $R^{A 1325 D}$ is $<0.001, P$-value between UAS-edem1-RNAi and UAS-InR ${ }^{A 1325 D}$ is 0.0038, P-value between UAS-InR $R^{A 1325 D}$ and UAS-edem1-RNAi, UAS-InR ${ }^{A 1325 D}$ is $<0.001$ and $P$-value between control and UAS-edem1-RNAi, UAS-InR $R^{A 1325 D}$ is $<0.001$ [log-rank test], Wald test $=10.53$ on $\mathrm{df}=1, P=0.001$ [Cox proportional hazard analysis]). (A, B, C', D) (P-value *<0.05; ${ }^{* *}<0.01,{ }^{* * *}<0.001$; Data information: In [A, B, C", D] data are presented as mean \pm SEM).

Source data are available for this figure.

levels in the fat body when compared to that of control (Fig 2 $\mathrm{C}^{\prime}$ and $C^{\prime \prime}$ ), which suggested an accumulation of DILP2 protein in the IPCS. Together, these observations suggest that edem1 function in the fat body maintains systemic insulin signalling in the larvae by the regulation of IPC activity.

As the next approach, we tested whether the reduction in insulin signalling in response to blocking edem 1 in the fat body was responsible for the metabolic phenotypes. A constitutively active form of insulin receptor $\left(I n R^{A 1325 D}\right)$ was co-expressed with edem1RNAi in the fat body. InR $R^{A 1325 D}$, which harbours an Ala-Asp mutation at position 1325, would activate downstream insulin signalling independent of DILP ligand and hence should alleviate phenotypes caused by low insulin signalling (Broughton et al, 2005; Tettweiler et al, 2005; DiAngelo \& Birnbaum, 2009; Kannan \& Fridell, 2013). As 
expected, expression of $I n R^{A 1325 D}$ was sufficient to alleviate high triglyceride levels and starvation resistance observed in response to knock down of edem1 levels in the fat body (Fig 2D and E). We have performed experiments using UAS-control transgenes to rule out the effect of Gal4 titration in this experiment and all future rescue experiments where multiple UAS transgenes are driven by the same Gal4 driver (data not shown). These experiments show that $\operatorname{In} R^{A 1325 D}$ expression in the fat body alleviated the decrease in insulin signalling, caused by blocking edem1, as shown by 4 ebp and dilp6 mRNA levels (Fig S1D' and D"'). Reduced dilp3 levels (Fig S1D") and accumulation of DILP2 caused by lowering edem1 levels in the fat body was rescued by $\operatorname{In} R^{A 1325 D}$ (Fig S1E).

Thus, blocking edem 1 in the fat body reduced systemic insulin signalling, which led to metabolic phenotypes. The rescue of phenotypes caused by lowering edem1 levels by the constitutively active form of InR could be due to an increase in insulin signalling in the fat body cells or could be due to an indirect effect at the level of IPCS. These experiments confirmed that Edem 1 function in the fat body is crucial to maintain systemic insulin signalling and metabolic homeostasis.

\section{Fat body-derived signals are involved in Edem1-mediated regulation of IPCS}

Drosophila fat body controls IPC function with the aid of a set of humoral factors, which relays the nutritional status of the organism to the IPCS. The fat body-derived signals (FDSs) control DILP release from the IPCs into the hemolymph leading to effects on growth and maintenance of metabolic balance. In addition, changes in dilp gene expression have also been reported in response to fat body-derived signals. We next investigated whether blocking Edem1 led to changes in the levels of FDSs and thereby the function of IPCS.

To test the role of FDSs in edem1 knock down phenotypes, we measured the levels or activity of various FDSs. We saw an increase in the transcript levels of dilp6 in response to knock down of edem1 in the fat body (Fig 2A'). Next, we measured the mRNA levels of upd2, totA, and the levels of STAT92E-GFP as readouts for activity of JAKSTAT pathway, a cell signalling pathway activated by Upd2, an FDS reported to regulate IPC functions (Rajan \& Perrimon, 2013). Blocking edem1 expression in the fat body led to a decrease in totA and upd 2 mRNA levels (Fig S2A' and A"). In addition, STAT92E-GFP expression in the brain was found to be low in response to reduced edem1 in the fat body (Fig S2B' and B"). We also measured Drosophila TNF $\alpha$ Eiger levels, another FDS that acts on IPCs through its receptor Grindelwald, and activation of downstream JNK signalling by Eiger. The transcript levels of tace, the TNF $\alpha$ converting enzyme encoding gene, eiger and nlaz, a key target of JNK signalling were measured (Hull-Thompson et al, 2009; Pasco \& Leopold, 2012; Agrawal et al, 2016), and the levels of these genes were found to be increased in response to edem1-RNAi (Fig 3A, B, and D). The cleaved form of Eiger protein (s-Egr) in the whole-body extracts was also found to be higher in edem1-RNAi (Fig 3 $C^{\prime}$ and $C^{\prime \prime}$ ), which confirmed enhanced levels of active form of Eiger released by blocking Edem1 function in the fat body. Eiger and Dilp6 are considered to be negative regulators of IPC function, whereas Upd2 is expected to activate IPCs (Bai et al, 2012; Rajan \& Perrimon, 2013; Agrawal et al,
2016), and the gene expression changes observed here suggested that these FDSs might mediate the effects of edem1 knock down on insulin signalling.

\section{Eiger is involved in Edem1-mediated regulation of IPC}

To identify the FDS(s) involved in mediating the metabolic phenotypes observed by blocking edem1 levels, we expressed eigerRNAi, dilp6-RNAi or upd2, together with edem1-RNAi in the fat body. Down-regulation of eiger mRNA and overexpression of upd2 rescued edem 1 knock down phenotypes of lipid stores and starvation resistance; however, there were no effects with dilp6-RNAi (Figs 3E and $F$ and S2D, E, G, and H). This suggested that either Upd2 or Eiger could be regulated by Edem 1 and manage IPC function. We did not see any significant changes to DILP2 levels in the IPCS, dilp3 mRNA levels and insulin target genes by overexpression of $u p d 2$ in fat body that express edem1-RNAi (Fig S2C and F). However, when we down-regulated eiger mRNA in edem1-RNAi expressing fat body, we observed a reduction in DILP2 puncta in the IPCs seen in response to edem1-RNAi expression in the fat body (Fig 4A' and A"). In addition, transcript levels of dilp3 (Fig 4B) and insulin target genes dilp6 (Fig 4C') and inr (Fig 4C') were restored by reducing Eiger levels in the edem1-RNAi background. Furthermore, the increase in glucose levels in the hemolymph seen in response to blocking edem1 was suppressed by co-expression of eiger-RNAi (Fig 4D). Thus, Edem1-mediated regulation of Eiger is crucial for managing insulin levels and nutrient homeostasis, by acting on the IPCS. Whereas, the Edem1-mediated regulation of Upd2 function also manages nutrient homeostasis, but does not do so by acting at the level of insulin signalling, suggesting an IPC-insulin signalling independent regulation of metabolic status by Edem1.

The fat body-derived cytokine Eiger is an upstream activator of c-Jun N-terminal kinase (JNK) pathway in flies and previous studies have shown that JNK signalling extends life span and limits growth by antagonizing cellular and organism-wide responses to insulin signalling (Hirosumi et al, 2002; Oh et al, 2005; Wang et al, 2005; Andersen et al, 2015; Agrawal et al, 2016). The increase in nlaz transcript levels in response to blocking edem1 levels in the fat body was rescued completely by the co-expression of eiger-RNAi, showing that the increase in JNK signalling in response to blocking edem1 expression in the fat body is due to enhanced Eiger levels (Fig 3D). These experiments confirm that Edem1 function in the fat body regulates Eiger activity and JNK signalling.

Next, we carried out experiments to confirm if regulation of Eiger by Edem1 is crucial for maintaining IPC function and metabolic homeostasis. Towards this we used two approaches: (i) we activated TOR signalling pathway in edem1-RNAi expressing fat body (Fig 5), as TOR signalling has been shown to block Eiger activation and (ii) we performed co-culture experiments by blocking the TNF receptor grindelwald in the IPCS (Fig 6). TOR signalling pathway regulates a plethora of cellular processes including cell growth, proliferation, cell survival, etc., depending on nutrient levels. Recently, TOR has been reported to repress tace transcription, which would in turn suppress the production of active Eiger from the fat body (Agrawal et al, 2016). Rheb (Ras homolog enriched in brain), a member of Ras superfamily of GTP binding proteins, activates TOR kinase and results in growth and regulation of metabolic pathways (Oldham et 
A

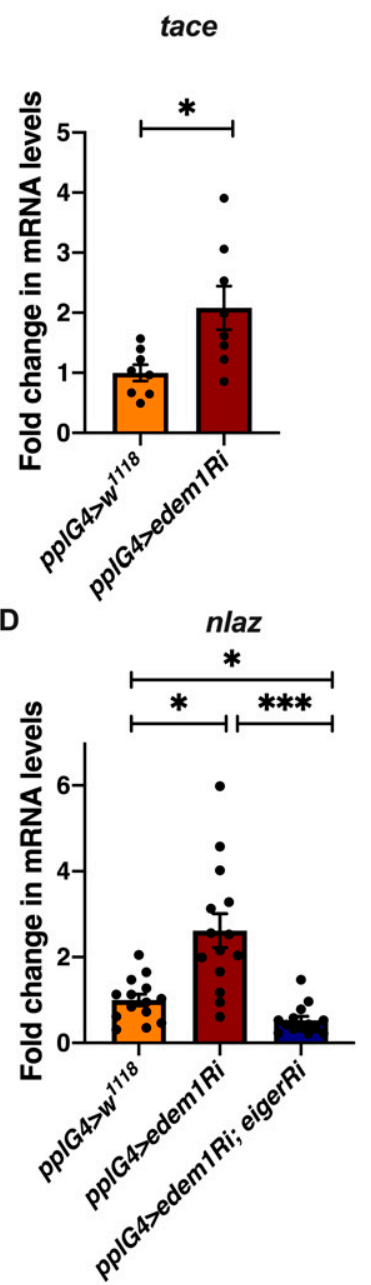

B

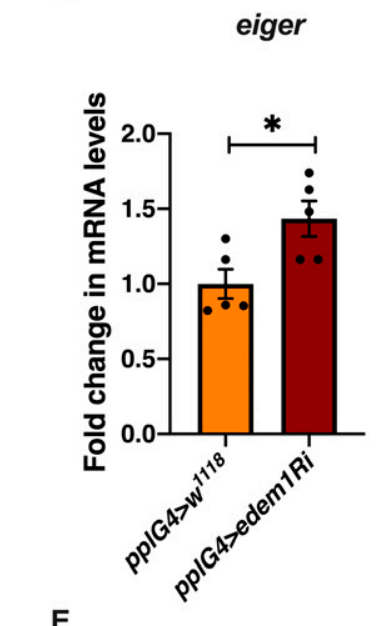

$E$

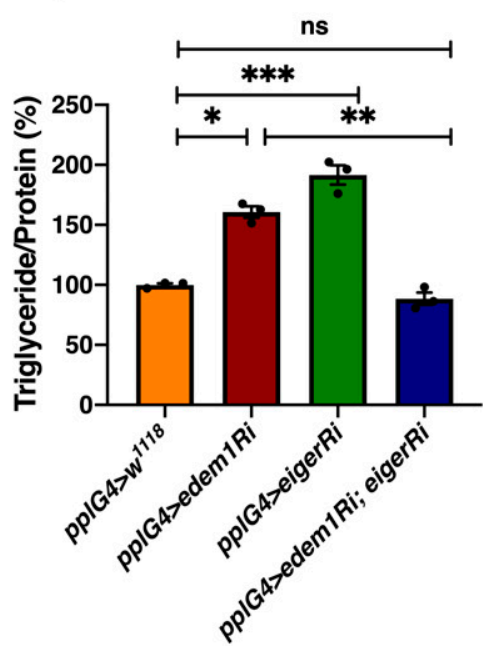

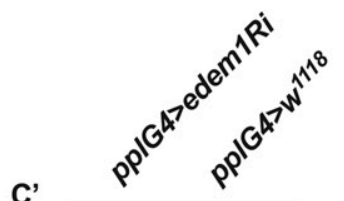

C'

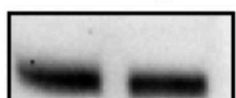

IB: FL - egr

C"

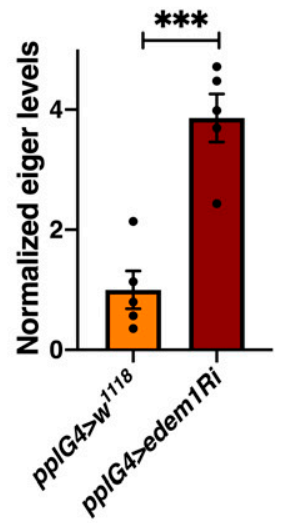

IB: s - egr



$\mathbf{F}$
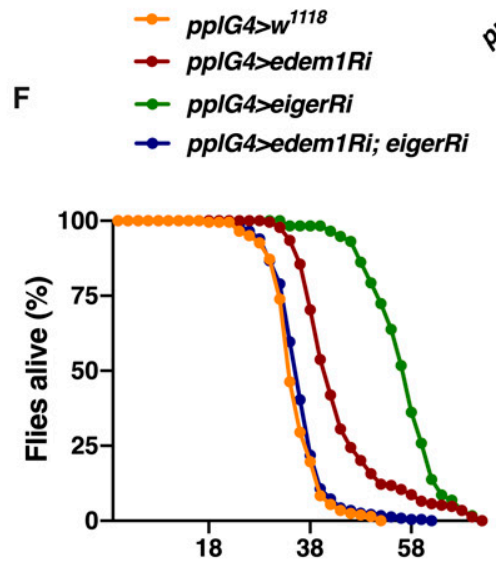

Hours of starvation

Figure 3. Reduction in edem 1 levels in the fat body enhanced JNK signalling.

(A) Blocking edem1 expression using RNAi in the fat body led to an increase in mRNA levels of tace. Data are shown as fold change in mRNA levels in third instar larvae, values are normalised to pplGal $4>W^{1118}$ and fold change in pplGal $4>U A S$-edem1-RNAi is shown (independent biological replicates $=8, P$-value between control and UASedem1-RNAi is 0.0211 [Welch's $t$ test]). (B) Blocking edem1 expression using RNAi in the fat body led to an increase in mRNA levels of eiger. Data are shown as fold change in mRNA levels in third instar larvae, values are normalised to $p p l G a l 4>W^{1118}$ and fold change in pplGal $>>U A S-e d e m 1-R N A i$ is shown (independent biological replicates $=5$, $P$-value between control and UAS-edem1-RNAi is 0.0226 [Welch's $t$ test]). (C) Blocking edem1 expression using RNAi in the fat body led to an increase in the circulating levels of eiger in 5-d-old male flies ( $C^{\prime}$ ). Data are shown as fold change in normalised soluble eiger levels $\left(C^{\prime \prime}\right)$, values are normalised to $p p l G a l 4>w^{1118}$, and fold change in pplGal4>UAS-edem1-RNAi is shown (independent biological replicates $=5, P$-value between control and UAS-edem1-RNAi is 0.0006 [Welch's $t$ test]). (D) Expression of eiger-RNAi in the fat body rescued increase in nlaz mRNA levels caused by blocking edem 1 levels in the fat body, and data are shown as fold change in mRNA levels. Data are shown as fold change in mRNA levels in third instar larvae, values are normalised to pplGal4>w1118 and fold change in pplGal4>UAS-edem1-RNAi, pplGal4>UAS-eigerRNAi and pplGal4>UAS-edem1-RNAi; UAS-eiger-RNAi is shown (independent biological replicates $=14$. $P$-value between control and UAS-edem1-RNAi is 0.0171 , $P$-value between UAS-edem1-RNAi and UAS-edem1-RNAi; UAS-eiger-RNAi is <0.001 and between control and UAS-edem1-RNAi; UAS-eiger-RNAi is 0.1085 [Kruskal-Wallis test followed by Dunn's post hoc test]). (E) Expression of eiger-RNAi in the fat body rescued increase in triglyceride caused by blocking edem 1 levels in the fat body. Data are shown as \% ratio of triglyceride to total protein levels in 5-d-old male flies, normalised to $100 \%$ in pplGal $>>w^{1118}$ (control), and changes in experimental conditions pplGal4>UAS-edem1-RNAi and pplGal4>UAS-edem1-RNAi; UAS-eiger-RNAi is shown (independent biological replicates $=3, P$-value between control and UAS-edem1-RNAi is 0.0052 , $P$-value between control and UAS-eiger-RNAi is $<0.001$, $P$-value between UAS-edem1-RNAi and UAS-edem1-RNAi, UAS-eiger-RNAi is $<0.001$, $P$-value between UASedem1-RNAi and UAS-eiger-RNAi is 0.8315, P-value between UAS-eiger-RNAi and UAS-edem1-RNAi, UAS-eiger-RNAi is <0.001 and P-value between control and UASedem1-RNAi, UAS-eiger-RNAi is >0.9999 [Kruskal-Wallis test followed by Dunn's post hoc test]). (F) Enhanced starvation resistance shown as percentage of flies ( $5 \mathrm{~d}$ old) which were alive at various time points of starvation in the following genotypes-pplGal $4>W^{1118}$, pplGal $4>U A S-e d e m 1-R N A i$, and pplGal4> UAS-edem1-RNAi; UAS-eigerRNAi-is shown (independent biological replicates $=3$, number of flies used for control is 203, for pplGal4>UAS-edem1-RNAi is 229, for pplGal $4>U A S-e i g e r-R N A i$ is 58 and for pplGal4> UAS-edem1-RNAi, UAS-leiger-RNAi is 233. P-value between control and UAS-edem1-RNAi is <0.001, P-value between control and UAS-eiger-RNAi is <0.001, $P$-value between UAS-edem1-RNAi and UAS-edem1-RNAi, UAS-eiger-RNAi is $<0.001, P$-value between UAS-edem1-RNAi and UAS-eiger-RNAi is $<0.001, P$-value between UAS-eiger-RNAi and UAS-edem1-RNAi, UAS-eiger-RNAi is $<0.001$ and $P$-value between control and UAS-edem1-RNAi, UAS-eiger-RNAi is 0.0459 [log-rank test], Wald test $=$ 
al, 2000; Garami et al, 2003; Saucedo, 2003; Oldham, 2011). Increase in the levels of eiger transcript levels and JNK pathway target nlaz in response to edem 1 down-regulation in the fat body was abrogated by overexpression of rheb (Fig $5 \mathrm{~A}$ ), which confirms that activating TOR signalling is sufficient to suppress JNK signalling possibly by the regulation of Eiger activity. Changes in the reduction in insulin signalling (Fig 5B), dilp3 transcript levels (Fig 5C), excess fat levels (Fig 5D), and starvation resistance (Fig 5E) in response to blocking Edem1 levels were rescued by the overexpression of rheb in the fat body. This confirmed that increasing TOR activity and thereby reducing the activation of JNK levels was sufficient to rescue the systemic insulin signalling and the metabolic phenotypes such as fat storage and starvation sensitivity.

Next, we tried to show that Edem1 function in the fat body regulates Eiger activity on IPCS. In low-protein diet, the soluble form of Eiger binds to its receptor Grindelwald (Grnd) in the IPCS, thereby activate JNK signalling and suppress dilp transcript levels (Andersen et al, 2015; Agrawal et al, 2016). We performed ex vivo organ culture experiments, using hemolymph isolated from control larvae and larvae in which edem1 expression in the fat body was blocked. Brains dissected from wild-type larvae and larvae with grnd knock down in the IPCS, were incubated with the hemolymph from the above-mentioned conditions. As expected, treatment of control larval brains with hemolymph from edem1-RNAi larvae (THCB) led to accumulation of DILP2 in the IPCS, when compared with hemolymph from control larvae (CHCB) (Fig 6A and B). We observed less DILP2 puncta in IPCs in response to blocking grnd (THTB) when compared with control IPCs treated with hemolymph from edem1-RNAi larvae (THCB) (Fig 6A and B). Blocking grnd did not change the levels of DILP2 in the IPCS as IPC>grnd-RNAi brains did not show any changes in DILP2 puncta when treated with hemolymph extracted from control larvae (CHTB). Thus, accumulation of DILP2 in the IPCs in response to blocking edem1 levels in the larval fat body is due to Eiger activity on the IPCs through TNF receptor Grindelwald. Together these results confirm that Edem1 activity in the fat body regulates Eiger-mediated JNK signalling in the IPCS and manage systemic insulin signalling and metabolic status of flies.

\section{Reduction in edem1 levels during starvation is crucial for survival}

Insulin signalling aids an organism to respond to changes in the nutrient environment by managing various biological functions. In response to nutrient deprivation insulin signalling is reduced, which would allow an organism to manage its energy stores and induce various hunger triggered behavioral responses (Britton et al, 2002; Arsic \& Guerin, 2008; Erion \& Sehgal, 2013). Our experiments show that lowering edem1 levels improved survival against starvation by reducing insulin signalling (Figs $1 \mathrm{C}$ and $2 \mathrm{E}$ ). Hence, we tested if edem1 levels are lowered during nutrient deprivation, which may aid in better survival of flies by the reduction in insulin levels. Moreover, blocking edem 1 in the fat body in larvae was sufficient to enhance the appetite, similar to hunger induced responses observed in food deprived larvae (Fig 1G). As expected, in response to food depletion we observed a reduction in edem1 mRNA levels (Fig 7A). To test whether reduction in edem1 levels is essential for protection against starvation, we overexpressed edem 1 in the fat body in food deprived flies and checked their starvation responses. Overexpression of edem 1 in the fat body reduced the survival of flies during food deprivation (Figs 7B' and $\mathrm{S} 3 \mathrm{~A}$ ), confirming that the reduction in edem1 levels is crucial for survival in response to nutrient depletion. Whereas overexpression of Edem 1 did not affect fat stores in the adult flies, higher levels of Edem 1 blocked utilisation of TGA in starved animals and caused enhanced mortality (Fig 7B). Thus, reduction in Edem1 during starvation facilitates mobilisation of stored fat, which aids in enhanced survival by meeting energy requirements during acute food deprivation. Higher Edem1 levels during starvation blocked the increase in eiger transcripts (Fig 7C), although the levels of the cleaved form of Eiger did not get affected either in fed or starved conditions. In response to acute starvation, JNK signalling is increased and the transcript levels of dilp3 and insulin signalling are decreased (Fig $7 \mathrm{C}-\mathrm{F}$ ). These effects of starvation were abrogated by edem1 overexpression in the fat body (Fig 7C-F). Meanwhile, overexpression of Edem1 in the fed conditions did not affect fat levels and insulin signalling. Thus, elevated Edem1 expression affects metabolic homeostasis and survival during starvation conditions. Here, we conclude that lowering of edem 1 transcripts in the fat body during starvation facilitates activation of Eiger and reduction in insulin signalling, which results in the enhanced survival of flies.

\section{Discussion}

Fluctuations in nutrient levels would trigger organism-wide changes, which includes alterations to various metabolic pathways. Changes in the metabolic pathways would aid the organism in managing the growth and maintenance of nutrient stores according to the availability of food. Apart from these biochemical changes, hunger elicits stereotypic behavioral responses, which includes an enhanced urge to feed, increased foraging, acceptance of unpalatable food, etc. (Chouhan et al, 2017). Several of the crucial responses such as mobilisation of stored nutrients and enhanced urge to feed, which aids the organism to survive nutrient deprivation is triggered by the reduction in systemic insulin signalling (Britton et al, 2002; Rulifson et al, 2002; Broughton et al, 2005; Tettweiler et al, 2005; Arsic \& Guerin, 2008; DiAngelo \& Birnbaum, 2009; Kannan \& Fridell, 2013). In Drosophila, IPCS respond to changes in the availability of food and modulate DILP levels under the control of the fat body, which acts as a nutrient sensor. Various fat body-derived signals act on IPCs directly or indirectly, and the regulation of these signals in response to changes in the nutrient status of Drosophila plays a key role in maintaining systemic insulin levels (Colombani et al, 2003; Géminard et al, 2009; Bai et al, 2012; Rajan \& Perrimon, 2012; Ghosh \& O'Connor,

0.09 on $\mathrm{df}=1, P=0.8$ [Cox proportional hazard analysis]). (A, B, C", D, E) $\left(P\right.$-value *<0.05; ** $<0.01,{ }^{* * *}<0.001$; Data information: In [A, B, C", D, E], data are presented as mean \pm SEM).

Source data are available for this figure. 


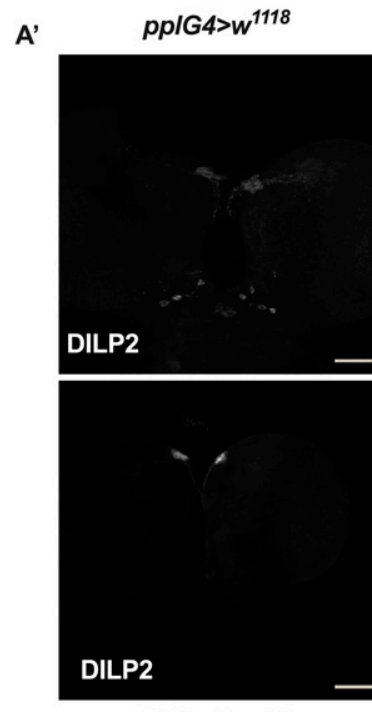

pp/G4>eigerRi
pp/G4>edem1Ri
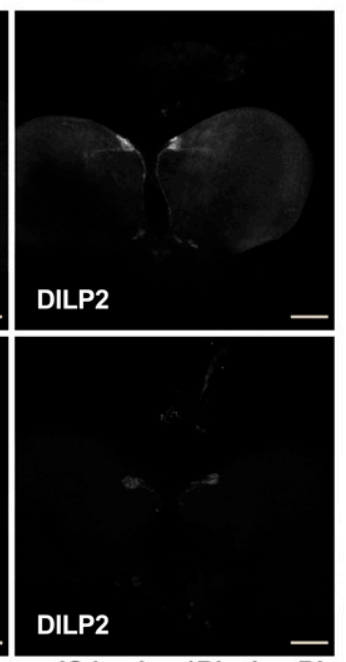

pp/G4>edem1Ri; eigerRi

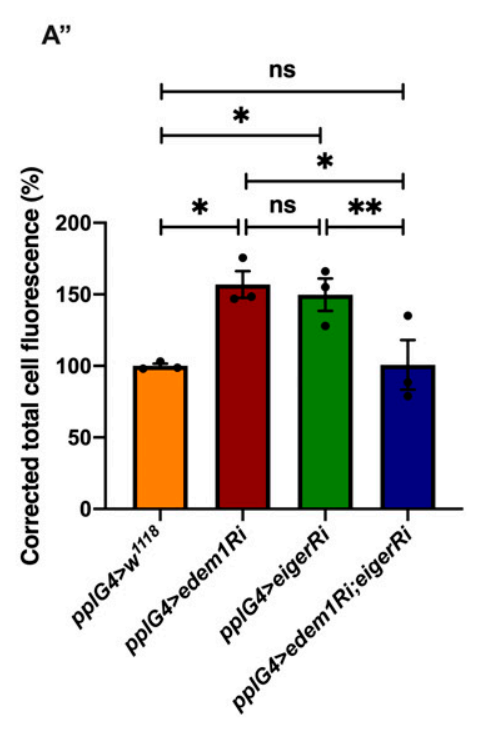

B
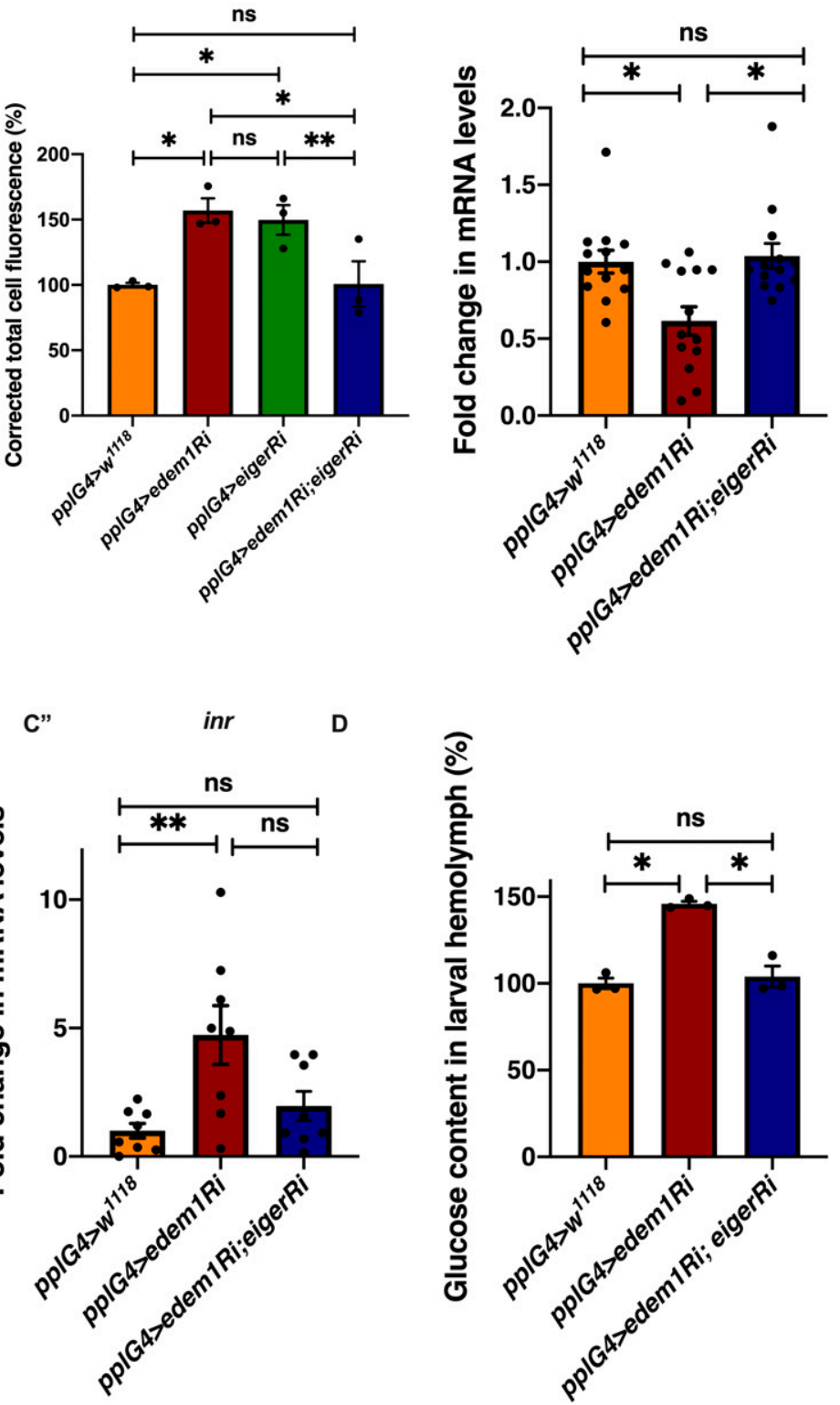

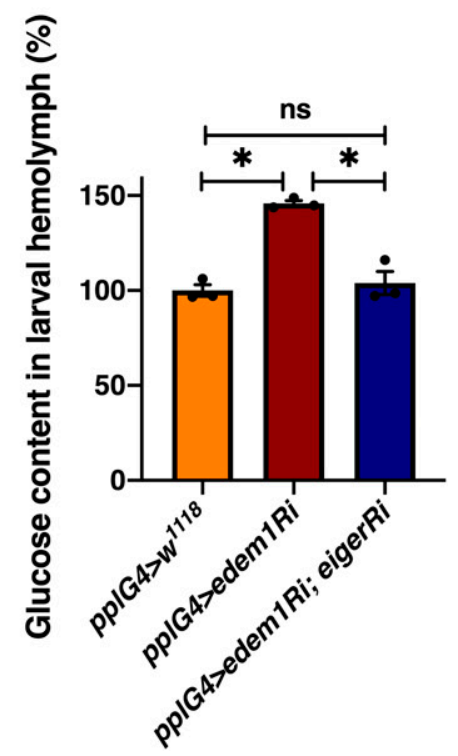

Figure 4. Knock down of eiger in the fat body rescued the metabolic phenotypes.

(A) DILP2 levels in the insulin-producing cells in response to edem1-RNAi was rescued by reducing eiger in the fat body ( $A^{\prime}$ ). Shown are representative images of anti-DILP2 antibody staining in larval brains of $p p l G a l 4>W^{1118}$ (independent biological replicates $=15$ ); $p$ plGal $4>U A S$-edem1-RNAi (independent biological replicates = 14); $p$ lGal $>$ UASeiger-RNAi (independent biological replicates $=19$ ) and pplGal4> UAS-edem1-RNAi; UAS-eiger-RNAi (independent biological replicates = 9). Corrected total cell fluorescence values are normalised to pplGal4>W ${ }^{1118}$ and fold change in pplGal4>UAS-edem1-RNAi, pplGal4>UAS-eiger-RNAi and pplGal4> UAS-edem1-RNAi; UAS-eiger-RNAi is shown in $\left(\mathrm{A}^{\prime \prime}\right)$ ( $P$-value between control and UAS-edem1-RNAi is 0.0444, $P$-value between control and UAS-eiger-RNAi is 0.0270, P-value between UAS-edem1-RNAi and UAS-eiger-RNAi is $0.9967 P$-value between control and UAS-edem1-RNAi; UAS-eiger-RNAi is >0.9999, $P$-value between UAS-edem1-RNAi and UAS-edem1-RNAi; UAS-eiger-RNAi is 0.0195 and P-value between UAS-eiger-RNAi and UAS-edem1-RNAi; UAS-eiger-RNAi is 0.0072 [Kruskal-Wallis test followed by Dunn's post hoc test]) (scales: 50 Mm). (B) Reduction of dilp3 mRNA levels, in response to edem1-RNAi was rescued by reducing eiger in the fat body, data is shown as fold change in mRNA levels in third instar larvae, values are normalised to pplGal4> $w^{1118}$ fold change in pplGal4>UAS-edem1-RNAi and pplGal4> UAS-edem1-RNAi; UAS-eiger-RNAi is shown (independent biological replicates = 13. P-value between control and UAS-edem1-RNAi is 0.0395, between control and UAS-edem1-RNAi; UAS-eiger-RNAi is >0.9999 and between UAS-edem1-RNAi and UAS-edem1-RNAi; UAS-eigerRNAi is 0.0253 [Kruskal-Wallis test followed by Dunn's post hoc test]). (C) Increase in dilp6, in response to edem1-RNAi was rescued by reducing eiger in the fat body ( $C^{\prime}$ ) and inr $\left(C^{\prime \prime}\right)$. Data are shown as fold change in mRNA levels in third instar larvae, values are normalised to pplGal $4>W^{1118}$ and fold change in pplGal4>UAS-edem1-RNAi and pplGal4> UAS-edem1-RNAi; UAS-eiger-RNAi is shown (independent biological replicates $=11$ for dilp6 and independent biological replicates $=8$ for inr. $P$-value between control and UAS-edem1-RNAi is 0.0392 for dilp6 and 0.0194 for inr. P-value between control and UAS-edem1-RNAi; UAS-eiger-RNAi is >0.9999 for dilp6 and >0.9999 for inr, P-value between UASedem1-RNAi and UAS-edem1-RNAi; UAS-eiger-RNAi is 0.0296 for dilp6 and 0.1976 for inr [Kruskal-Wallis test followed by Dunn's post hoc test]). (D) Increase in Glucose levels in the hemolymph in response to edem1-RNAi was rescued by reducing eiger in the fat body. Data are shown as \% of glucose levels in the larval hemolymph, normalised to $100 \%$ in pplGal4>W ${ }^{1118}$ (control) and changes in experimental conditions pplGal4>UAS-edem1-RNAi and pplGal4> UAS-edem1-RNAi; UAS-eiger-RNAi is shown (independent biological replicates $=3, P$-value between control and UAS-edem1-RNAi is 0.0105, P-value between control and UAS-edem1-RNAi; UAS-eiger-RNAi is $>0.9999$, $P$-value between edem1-RNAi and UAS-edem1-RNAi; UAS-eiger-RNAi is 0.0175 [Kruskal-Wallis test followed by Dunn's post hoc test]). (A", B, C, D) $(P$-value *<0.05; ** $<0.01$, *** $<0.001$; Data information: In [A", $B, C, D]$, data are presented as mean \pm SEM).

Source data are available for this figure. 

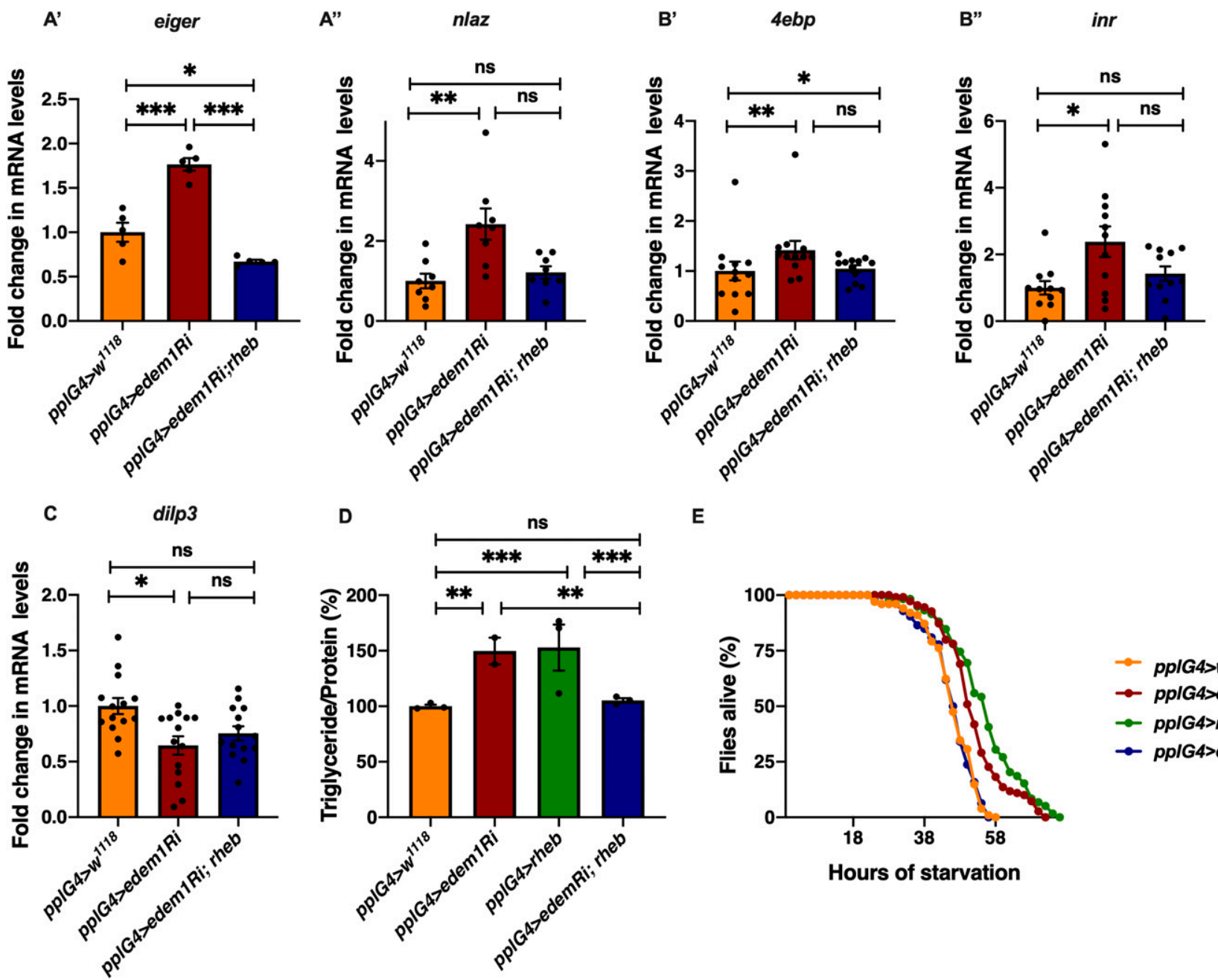

E

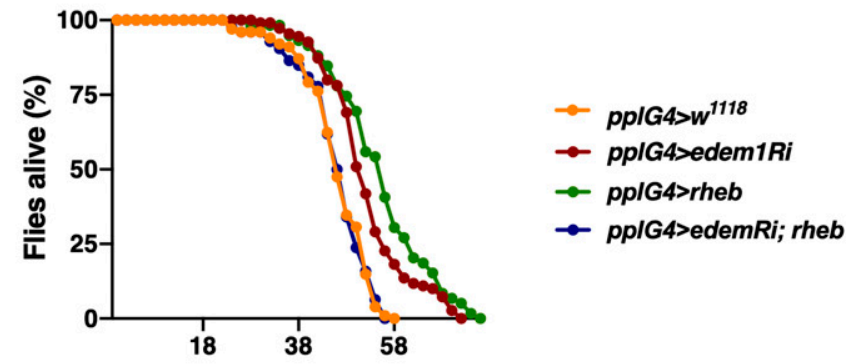

Hours of starvation

Figure 5. Activating target of rapamycin signalling rescued edem1-mediated phenotypes.

(A) Increase in mRNA levels of eiger (A') and nlaz (A") in response to blocking edem1 expression was rescued by co-expression of UAS-rheb. Data are shown as fold change in mRNA levels in third instar larvae, values are normalised to pplGal $4>W^{1118}$ and fold change in pplGal4>UAS-edem1-RNAi and pplGal $>>$ UAS-edem1-RNAi; UASrheb is shown (independent biological replicates $=5$ for eiger and 8 for nlaz. P-value between control and UAS-edem1-RNAi is $<0.0001, P$-value between UAS-edem1-RNAi and UAS-edem1-RNAi; UAS-rheb is $<0.0001$, P-value between control and UAS-edem1-RNAi; UAS-rheb is 0.0228 for eiger [ordinary one-way ANOVA followed by Tukey's post hoc test]. P-value between control and UAS-edem1-RNAi is 0.0039, P-value between UAS-edem1-RNAi and UAS-edem1-RNAi; UAS-rheb is $0.0486, P$-value between control and UAS-edem1-RNAi; UAS-rheb is >0.9999 for nlaz (Kruskal-Wallis test followed by Dunn's post hoc test]). (B) Increase in mRNA levels of 4ebp (B') and inr (B") in response to blocking edem1 expression was alleviated by co-expression of UAS-rheb. Data are shown as fold change in mRNA levels in third instar larvae, values are normalised to pplGal $>>W^{1118}$ and fold change in pplGal4>UAS-edem1-RNAi and pplGal $>>$ UAS-edem1-RNAi; UAS-rheb is shown (independent biological replicates $=12$ for 4 ebp. P-value between control and edem1-RNAi is $0.0085, P$-value between UAS-edem1-RNAi and UAS-edem1-RNAi; UAS-rheb is $0.1143, P$-value between control and UASedem1-RNAi; UAS-rheb is $>0.9999$ [Kruskal-Wallis test followed by Dunn's post hoc test]; independent biological replicates $=11$ for inr. P-value between control and UASedem1-RNAi is 0.0406, P-value between UAS-edem1-RNAi and UAS-edem1-RNAi; UAS-rheb is 0.7277, P-value between control and UAS-edem1-RNAi; UAS-rheb is 0.5799 [Kruskal-Wallis test followed by Dunn's post hoc test]). (C) Decrease in mRNA levels of dilp3 in response to blocking edem1 expression was rescued by co-expression of UAS-rheb. Data are shown as fold change in mRNA levels in third instar larvae, values are normalised to pplGal $4>W^{1118}$ and fold change in pplGal $4>\cup A S-e d e m 1-R N A i$ and pplGal4> UAS-edem1-RNAi; UAS-rheb is shown (independent biological replicates $=14$. P-value between control and UAS-edem1-RNAi is 0.0231, P-value between UASedem1-RNAi and UAS-edem1-RNAi; UAS-rheb is >0.9999, P-value between control and UAS-edem1-RNAi; UAS-rheb is 0.1356 [Kruskal-Wallis test followed by Dunn's post hoc test]). (D) Overexpression of rheb in the fat body rescued enhanced stored fat levels. Data are shown as \% ratio of triglyceride to total protein levels, in 5-d-old male flies, values are normalised to pplGal4>w1118 and fold change in pplGal4>UAS-edem1-RNAi and pplGal4> UAS-edem1-RNAi; UAS-rheb is shown (independent biological replicates $=4, P$-value between control and UAS-edem1-RNAi is 0.0003, P-value between control and UAS-rheb is $<0.001, P$-value between UAS-edem1-RNAi and UASedem1-RNAi, UAS-rheb is 0.0005 , P-value between UAS-edem1-RNAi and UAS-rheb is $0.5184, P$-value between UAS-rheb and UAS-edem1-RNAi, UAS-rheb is $<0.001$ and $P$-value between control and UAS-edem1-RNAi, UAS-rheb is $>0.9999$ [Kruskal-Wallis test followed by Dunn's post hoc test]). (E) Overexpression of rheb in the fat body rescued increased starvation resistance. Data are shown as percentage of 5-d-old male flies which were alive at various time points of starvation in the following

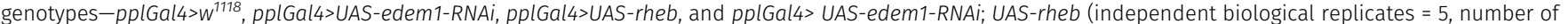
flies used for control is 101, for pplGal4>UAS-edem1-RNAi is 110, for pplGal4>UAS-rheb is 59 and for pplGal4> UAS-edem1-RNAi, UAS-rheb is 126. P-value between control and UAS-edem1-RNAi is $<0.0001$, $P$-value between control and UAS-edem1-RNAi is $<0.001$, $P$-value between control and UAS-rheb is $<0.001$, $P$-value between UAS-edem1RNAi and UAS-edem1-RNAi, UAS-rheb is $<0.001, P$-value between UAS-edem1-RNAi and UAS-rheb is $0.0247, P$-value between UAS-rheb and UAS-edem1-RNAi, UAS-rheb is $<0.001$ and $P$-value between control and UAS-edem1-RNAi, UAS-rheb is 0.9628 [log-rank test], Wald test $=0.21$ on $\mathrm{df}=1, P=0.6$ [Cox proportional hazard analysis]). $(A, B, C, D)\left(P\right.$-value ${ }^{*}<0.05 ;{ }^{* *}<0.01,{ }^{* \star *}<0.001$; Data information: In [A, B, C, D], data are presented as mean \pm SEM). Source data are available for this figure. 
A
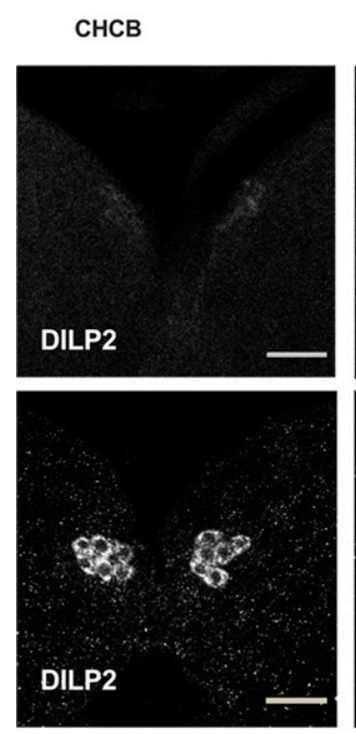

THCB
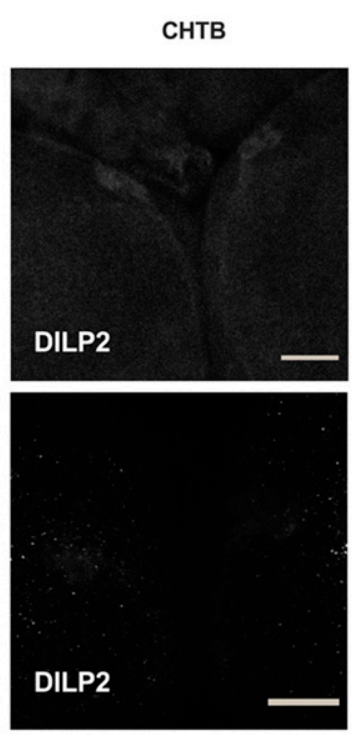

THTB
B

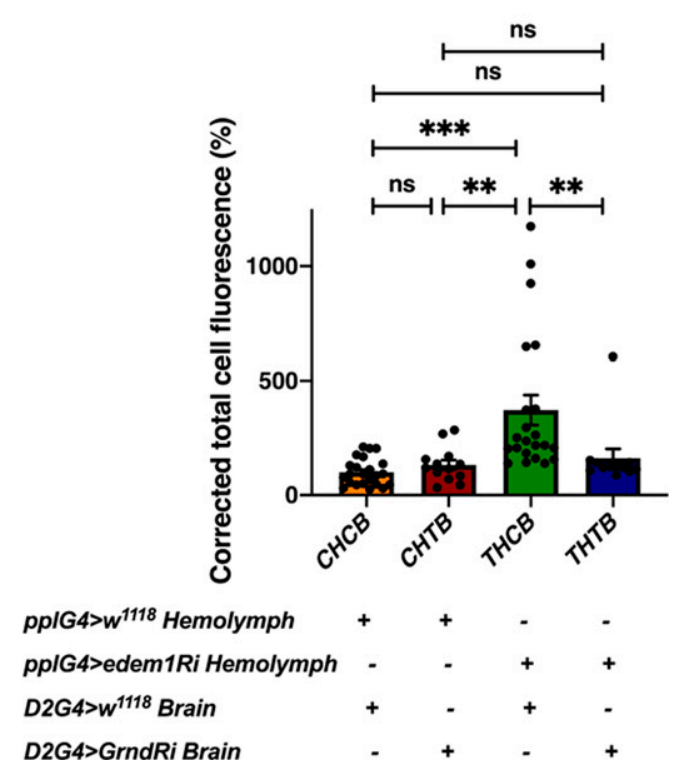

Figure 6. Blocking grindelwald in the insulin-producing cells (IPCS) rescued the increased accumulation of DILP2.

(A) DILP2 accumulation in the IPCS in response to edem1-RNAi was rescued by blocking Eiger receptor grnd in the IPCs. Representative images of anti-DILP2 antibody staining in larval brains of Dilp2Gal $>>W^{1118}$ treated with hemolymph from pplGal $4>W^{1118}$ (independent biological replicates $=23$ ) (CHCB), Dilp2Gal $4>U A S-g r n d-R N A i$ treated with hemolymph from pplGal4>W1118 (independent biological replicates $=13)(C H T B)$, Dilp2Gal $4>w^{1118}$ incubated with hemolymph from pplGal4>UAS-edem1-RNAi (independent biological replicates $=22)(T H C B)$ and Dilp2Gal4>UAS-grnd-RNAi incubated with hemolymph from pplGal4>UAS-edem1-RNAi (independent biological replicates $=12)($ THTB) larvae are shown $($ scales: $30 \mu \mathrm{m})$. $(B)$ Corrected total cell fluorescence values are normalised to CHCB and fold change in CHTB, THCB, and THTB are shown ( $P$-value between CHCB and CHTB is $>0.9999, P$-value between CHCB and THCB is $<0.001, P$-value between CHCB and THTB is $>0.9999$, $P$-value between $C H T B$ and THCB is $0.0018, P$-value between CHTB and THTB is $>0.9999$ and P-value between THCB and THTB is 0.0085 [Kruskal-Wallis test followed by Dunn's post hoc test]).

(B) $\left(P\right.$-value ${ }^{*}<0.05$; ${ }^{* *}<0.01,{ }^{* * *}<0.001$; Data information: In [B], data are presented as mean \pm SEM).

Source data are available for this figure.

2014; Agrawal et al, 2016; Delanoue et al, 2016; Droujinine \& Perrimon, 2016; Koyama \& Mirth, 2016; Sun et al, 2017). Here, we report a novel means by which the activity of a fat body-derived signal on IPCs is regulated.

While investigating the mechanisms that function in the fat body to control Drosophila IPCS, we identified Edem1, an ER-resident protein involved in ERAD mediated protein quality control. Edem 1 in the fat body maintains the activity of Drosophila TNF $\alpha$ Eiger (Fig 8A and B) and controls JNK signalling (Fig 8 C), thereby promoting normal IPC function (Fig 8D), maintain systemic insulin signalling (Fig 8E-G) and metabolic homeostasis (Figs 3 and 4). Eiger is activated by TACE, which cleaves the transmembrane form of Eiger and releases a soluble active form of Eiger into the hemolymph (Agrawal et al, 2016). TOR kinase, a key nutrient sensor, has been reported to control tace transcript levels and thereby Eiger activation. During low-protein diets, because of reduced TOR signalling, fat body releases the soluble form of Eiger, which would act on IPCS and activate JNK signalling to regulate dilp gene expression. Here, we identify Edem1 as a regulator of Eiger through the control of eiger and tace gene expression (Fig 3). We also show that activation of TOR signalling blocked the effects of suppression of edem 1 levels in the fat body (Fig 5), substantiating the role of Edem1 in regulating Eiger activity. At the moment, it is not clear if the TOR pathway acts through Edem1 to regulate eiger and tace gene expression, thereby manage Eiger activity. More efforts are also needed to identify the exact molecular mechanism by which Edem1 regulates Eiger.
We also found that the levels of Upd2, another FDS, are regulated by Edem1 function in the fat body. The fact that Upd2OE in edem1Ri background does not rescue the DILP2 accumulation and mRNA levels of dilp3 and other insulin targets suggests that Upd2 may act through another tissue unknown at the moment to regulate nutrient homeostasis. In support of this, we saw a decrease in tota in the whole larval RNA extracts and STAT92E-GFP levels in the entire brain in response to blocking edem 1 in the fat body (Figs S2A' and $B$ and 2). Hence, Edem1 function in the fat body acts in an IPC independent manner to maintain metabolic status. Here too, the exact molecular mechanism by which Edem1 regulates upd2 transcript levels is not understood at the moment. Although our experiments rule out the role of Upd2 in regulating insulin signalling in response to edem1-RNAi, we do not know how enhancement of upd2 levels in response to down-regulation of Edem1 in the fat body leads to IPC independent regulation of metabolic status.

Edem1 function in the fat body maintains systemic insulin signalling, and reduction in edem 1 levels in the fat body resulted in low systemic insulin signalling in larvae, which led to metabolic phenotypes as seen on circulating sugar levels and enhanced feeding in larvae; lipid and glycogen stores enhanced resistance to starvation and increase in life span in adult flies (Fig 1). Low insulin signalling has been reported to cause these phenotypes by previous studies (Böhni et al, 1999; Tatar et al, 2001; Rulifson et al, 2002; Broughton et al, 2005; Shingleton et al, 2005; Wu et al, 2005; Teleman et al, 2006; Slaidina et al, 2009; Grönke et al, 2010; Haselton et al, 

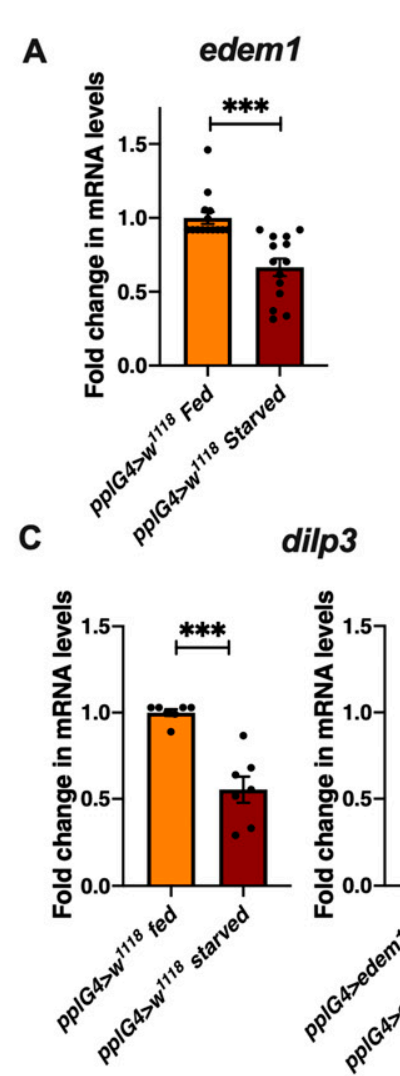

D"'

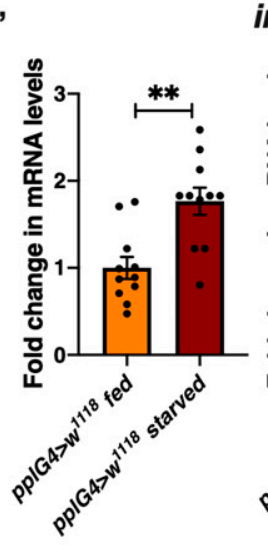

inr

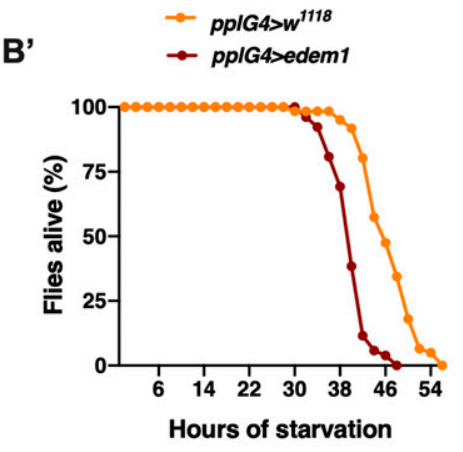

Hours of starvation

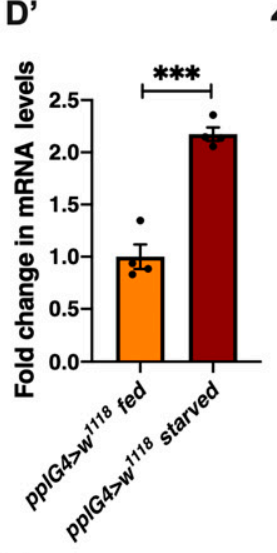

E
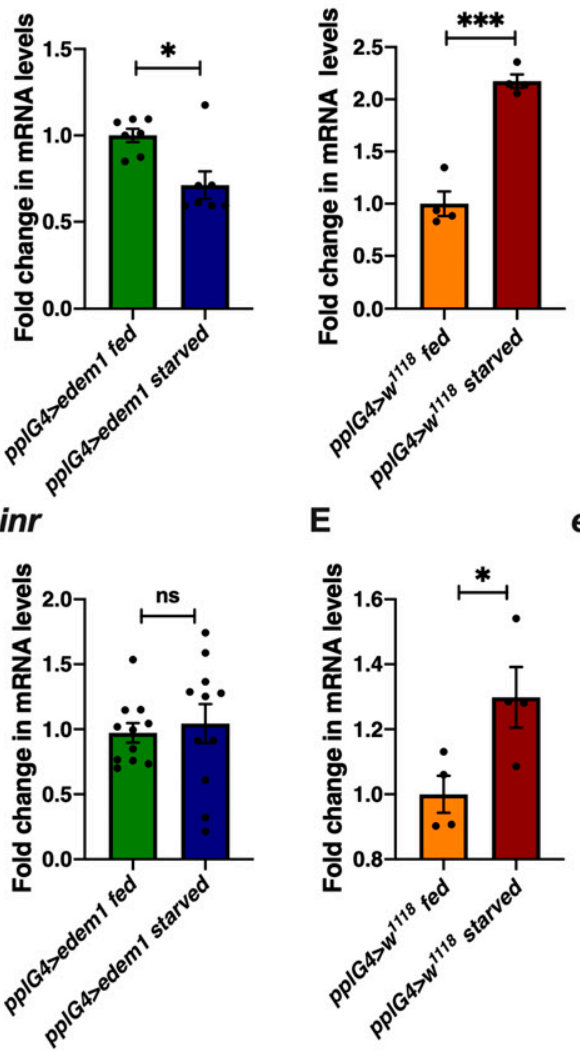

B"
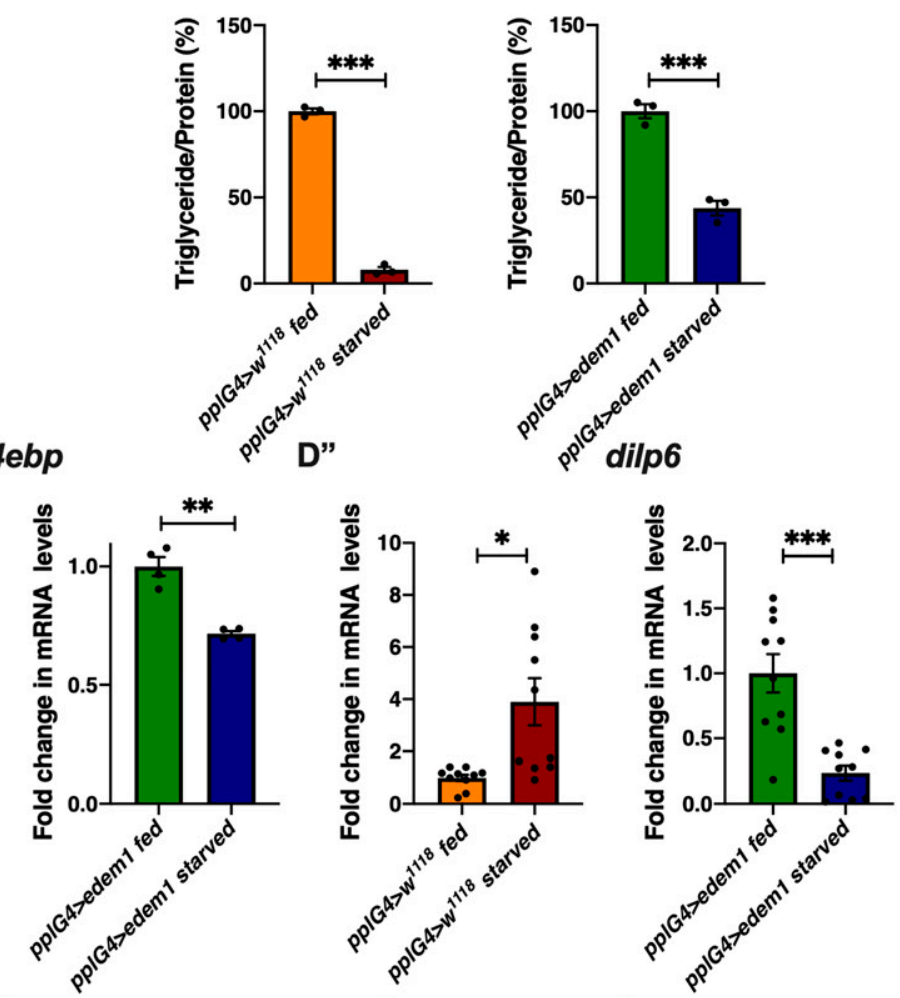

eiger

$$
F
$$

nlaz
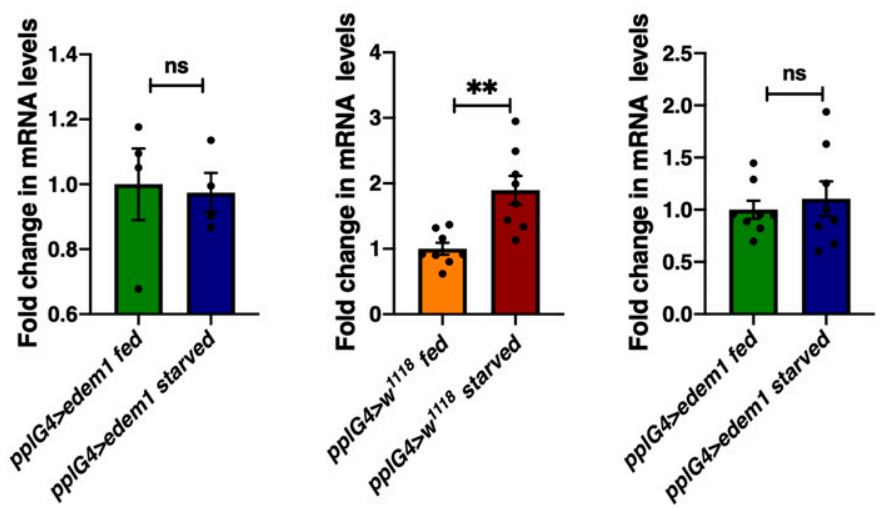

Figure 7. Reduction in edem1 levels during starvation is crucial for survival.

(A) Fold change in the mRNA levels of edem1 upon starvation in pplGal $>>W^{1118}$ larvae. Values are normalised to $p p l G a l 4>w^{1118}$ fed control and changes in the control starved are shown (independent biological replicates $=14, P$-value between control fed and starved larvae is $<0.0001$ [Mann-Whitney test]). (B) Overexpression of edem1 leads to enhanced sensitivity to starvation $\left(B^{\prime}\right)$, shown are percentage of male flies which were alive at various time points of starvation in the following genotypes-pplGal $4>w^{1118}$ and pplGal4>UAS-edem1 (independent biological replicates $=3$, number of flies used for control is 74 , for pplGal4>UAS-edem1 is 69 . P-value between control and UAS-edem 1 is $<0.0001$ [log-rank test], Wald test $=20.67 \mathrm{on} \mathrm{df}=1, P<0.001$ [Cox proportional hazard analysis]). (B") shows percentage reduction in triglyceride levels upon starvation. Data are shown as \% ratio of triglyceride to total protein levels, values are normalised to $p p l$ Gal $4>w^{1118}$ fed and fold change in pplGal $>>w^{1118}$ starved is shown (left) and values are normalised to pplGal4>UAS-edem1 fed and fold change in pplGal $4>U A S$-edem1 starved is shown (right) (independent biological replicates $=3$, P-value between pplGal $>>w^{1118}$ fed and pplGal $4>w^{1118}$ starved is $<0.0001$; P-value between pplGal $4>U A S$-edem1 fed and pplGal $4>$ UAS-edem 1 starved is $<0.0001$ Welch's $t$ test) (C) Overexpression of edem 1 in the fat body decreased the dilp3 mRNA levels when subjected to starvation. Data are shown as fold change in mRNA levels, values are normalised to pplGal $4>w^{1118}$ fed and fold change in pplGal $>>w^{1118}$ starved is shown (left) and values are normalised to pplGal4>UAS-edem1 fed and fold change in pplGal4>UAS-edem1 starved is shown (right) (independent biological replicates $=7$. P-value between control fed and starved is <0.001. P-value between UAS-edem1 fed and UAS-edem1 starved is 0.0251 [Mann-Whitney test]). (D) Overexpression of edem 1 in the fat body led to decreased levels of 4ebp ( $\left.\mathrm{D}^{\prime}\right)$, dilp6 ( $\left.\mathrm{D}^{\prime \prime}\right)$ and no change in inr ( $\left.\mathrm{D}^{\prime \prime \prime}\right)$ when subjected to starvation. Data are shown as fold change in mRNA levels, values are normalised to pplGal4>W1118 fed

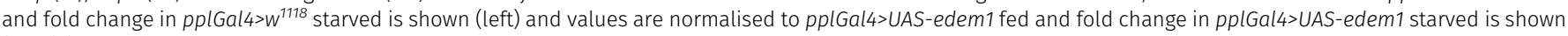
(right) (independent biological replicates $=4$ for $4 \mathrm{ebp}$, independent biological replicates $=10$ for dilp6 and independent biological replicates $=11$ for inr. $P$-value between control fed and starved is < 0.001 for 4 ebp, 0.0107 for dilp6, and 0.0012 for inr. P-value between UAS-edem 1 fed and UAS-edem 1 starved is 0.0039 for 4 ebp, < 0.001 for dilp6 and 0.6788 for inr [Welch's $t$ test]). (E) Overexpression of edem 1 in the fat body did not affect the levels of eiger mRNA when subjected to starvation. Data are shown as fold change in mRNA levels, values are normalised to pplGal $4>w^{1118}$ fed and fold change in pplGal $4>W^{1118}$ starved is shown (left) and values are normalised to 


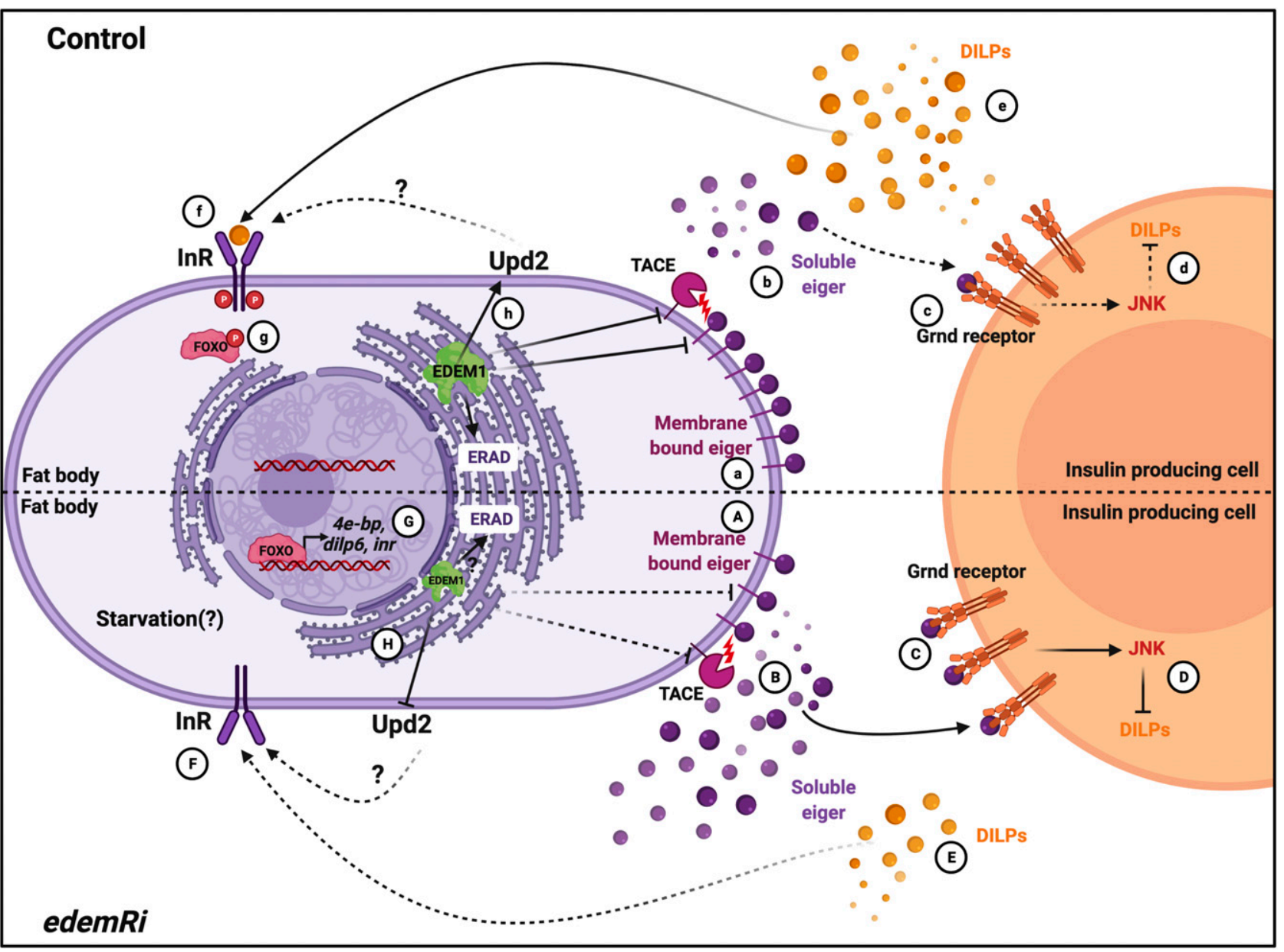

Figure 8. Working model.

In control and fed conditions, Edem1 blocks tace and eiger gene expression (a) and inhibits Eiger release (b) and as JNK signalling is kept low in the insulin-producing cells (c). This maintains insulin signalling (d-g). In edem1Ri background, tace and eiger gene expression is increased (A) and leads to increased Eiger secretion into the hemolymph (B). Soluble Eiger binds to the Grnd receptors in the insulin-producing cells and activate JNK signalling which inhibits insulin signalling (C and D). Reduction in insulin signalling mediated by reduction in Edem1 in the fat body (E, F, G) aid in survival of flies. Edem maintains Upd2 levels in the organisms ( $h$ ) which maintain the metabolic homeostasis, but in edem1Ri background Upd2 levels decrease (H) resulting in metabolic phenotypes (created with BioRender.com).

2010; Varghese et al, 2010; Bai et al, 2012; Hong et al, 2012). We also show that the impact of reducing edem 1 levels on insulin signalling is due to the accumulation of DILP2 protein in the IPCS (Fig 2C) and reduced dilp3 transcript levels in the larval IPCs (Fig 2B). However, it should be noted that we did not observe any changes at the protein and mRNA levels of other mNSC DILPS. DILPS are known to be regulated in a context specific manner, gene expression as well as protein levels in IPCs vary based on nutritional cues, developmental stages and various neural and endocrine signals that act on the IPCs (Ikeya et al, 2002; Grönke et al, 2010; Varghese et al, 2010; Hong et al, 2012; Söderberg et al, 2012; Luo et al, 2014; Kim \& Neufeld, 2015; Hallier et al, 2016). Eiger activity on IPCs in response to low protein diet has been shown to suppress dilp2 and dilp5 transcript levels (Agrawal et al, 2016). However, here we show that enhanced Eiger levels (Fig 8A and B) due to suppression of Edem1 expression in fed conditions affects dilp3 transcription and DILP2 protein accumulation in the IPCS (Fig 8C-E). Strictly the roles of individual DILPS are not understood; however, the effects of ablating IPCS, on growth and metabolism could be rescued by DILP2 expression alone (Rulifson et al, 2002; Haselton et al, 2010). Many reports hint at

pplGal4>UAS-edem1 fed and fold change in pplGal4>UAS-edem1 starved is shown (right) (independent biological replicates $=4$. $P$-value between control fed and starved is 0.0417. P-value between UAS-edem1 fed and UAS-edem1 starved is 0.8486 [Welch's $t$ test]). (F) Overexpression of edem1 in the fat body did not affect the levels of nlaz mRNA when subjected to starvation. Data are shown as fold change in mRNA levels, values are normalised to $p p l G a l 4>w^{1118}$ fed and fold change in $p p l G a l 4>w^{1118}$ starved is shown (left) and values are normalised to pplGal4>UAS-edem1 fed and fold change in pplGal4>UAS-edem1 starved is shown (right) (independent biological replicates $=8$. P-value between control fed and starved is 0.004 . $P$-value between UAS-edem $1 \mathrm{fed}$ and UAS-edem1 starved is 0.5887 [Welch's $t$ test]). (A, B", C, D, E, F) ( $P$-value ${ }^{*}<0.05$; ${ }^{* *}<0.01,{ }^{* * *}<0.001$; Data information: In [A, B", C, D, E, F], data are presented as mean \pm SEM).

Source data are available for this figure. 
effects on insulin signalling caused by an individual DILP or more than one DILP (Bai et al, 2012; Sudhakar et al, 2019).

Here, it may be noted that reduction in Edem1 levels in the fat body did not cause any effects on larval growth or developmental delays. One possible explanation for the lack of growth and developmental phenotypes is that the reduction in insulin signalling is not too drastic in response to lowering Edem1 levels in the fat body (Fig 2), in comparison to what is observed in response to insulin signalling pathway mutants or ablation of IPCs. However, the single and double mutants of both dilp2 and dilp3 produced very mild growth phenotypes (Grönke et al, 2010). Here, we report changes in the levels of dilp3 transcripts and DILP2 accumulation in the IPCs, which leads to no effects on body size, unlike complete loss of IPC function. Also, minimal reduction in insulin signalling in an inr mutant background showed elevated lipid and glycogen levels, whereas showing no effects on body size (Shingleton et al, 2005). Another possibility is the fact that the expression of edem1RNAi in the fat body enhanced feeding responses (Fig 1G), which may compensate for the growth effects caused by reduced insulin levels.

As our genetic screen and characterization of Edem1 was performed using the pplGal4 driver, which could be active in other tissues as well, we performed key experiments using an additional fat body driver CgGal4. We observed similar effects with both the Gal4 drivers, conforming that the effects we see with knocking down Edem1 is specific to fat body (Fig S4).

Managing insulin signalling during nutrient withdrawal is crucial for mobilisation of nutrient stores and survival. In response to starvation, we report that edem1 transcripts are reduced (Fig 7A). Reduction in edem 1 transcripts during starvation enhances eiger and nlaz levels, which aids in lowering insulin signalling (Fig $7 \mathrm{C}-\mathrm{F}$ ). This helps the flies to survive acute nutrient deprivation by mobilising energy stores (Figs 7B and 8). Surprisingly, we did not see any decrease in the levels of cleaved form of Eiger by enhancing Edem1 levels in the fat body. The levels of s-Egr are very low in normal fed flies (Fig 3C) and even during starvation, we did not see any increase as expected in s-Egr levels, although edem1 transcript levels are reduced. However, in response to starvation, we observe an increase in eiger transcript levels, which is attenuated by Edem1 overexpression. There is a concurrent change to nlaz levels during starvation, indicating an increase in JNK signalling, which is missing in Edem1 overexpressing flies. Hence, although there is significant Eiger processing in the context of reduced Edem1 levels in the fat body, in response to starvation, we are unable to document this and an increase in Eiger transcript levels seems to be the primary effect, which is rescued by Edem1 overexpression.

In edem1-RNAi flies, the triglyceride content of the flies is significantly higher as compared to controls, which makes them resist starvation as compared to their control counterparts. Whereas, flies with Edem1 overexpression when starved are unable to use triglyceride stores and hence died faster (Fig 7B). Thus, reduction of Edem1 levels plays an important role in eliciting survival responses to starvation and enhancing Edem 1 levels affected this, probably because of a failure in reducing insulin signalling and triglyceride mobilisation (Fig 7B-D). Moreover, reducing Edem1 levels in fed conditions led to enhanced feeding responses, similar to starvation conditions, further suggesting an active role for Edem1 in survival against food deprivation (Fig 1G). However, it is not yet clear if the function of Edem1 in regulating Eiger activity in the IPCS and systemic insulin signalling has any links to the ER stress pathway. Reduced edem 1 levels during starvation could be an outcome of reduced ER stress in response to low protein synthesis. Furthermore, reduction in edem 1 could cause aggregation of misfolded proteins in the $\mathrm{ER}$, which might be responsible for the changes we report here on Eiger levels. However, blocking few other essential components of ERAD mechanism did not give us any expected results (Fig S3B). Moreover, we did not observe any evidence for enhanced activity in the ER stress pathway in response to Edem1 down-regulation in the fat body. Hence, we are currently not sure whether Edem 1 activity on managing systemic insulin signalling and nutrient homeostasis is linked to its ERAD functions. We also cannot completely rule out a direct effect of Edem1 in the fat body on metabolic pathways, which is responsible for the nutrient homeostasis defects we report here.

To summarize, we show that Edem1, a key ERAD regulator, aids in the maintenance of nutrient homeostasis by managing the activity of TNF $\alpha$ Eiger on Drosophila insulin-producing cells (Fig 8). During fed conditions, Edem1 suppresses Eiger levels, which allows optimal insulin signalling and maintain a steady metabolic status. In response to starvation, our data suggest that lower levels of Edem1 leads to a reduction in insulin signalling and mobilisation of energy reserves, which aids in survival during acute food deprivation.

\section{Materials and Methods}

\section{Fly strains}

Fly stocks were reared in vials with standard food which consisted of $5.8 \%$ cornmeal, $5 \%$ dextrose, $2.36 \%$ yeast, $0.8 \%$ agar, and $10 \%$ Nipagen in $100 \%$ ethanol. All the flies were maintained at $25^{\circ} \mathrm{C}$ with 12:12 h light:dark cycle. CgGal4 (RRID:BDSC_7011), UAS-InR ${ }^{A 1325 D}$ (RRID:BDSC_8263), and UAS-edem1-RNAi (RRID:BDSC_58298) were obtained from Bloomington Drosophila stock center (BDSC). The RNAi lines used were obtained from Vienna Drosophila resource center (VDRC): UAS-edem1-RNAi (stock \#6923, 6922), UAS-eiger-RNAi (stock \#45253), UAS-grnd-RNAi (stock \#43454), UAS-dilp6-RNAi (GD) (stock \#45218), UAS-herp-RNAi (stock \#11724, 11725), and UAS-sip3RNAi (stock \#6870, 107060). dilp2-Gal4/CyOGFP, pumpless-Gal4, and $w^{1118}$ were obtained from Stephen Cohen. UAS-dEDEM1 was from Koichi lijima, UAS-rheb was obtained from Jagat. K. Roy, and UASupd2-EGFP/TM3Sb and 10XSTAT92E-GFP were obtained from Akhila Rajan. To match the genetic background all the fly strains used in this study were back-crossed into an isogenic $w^{1118}$ background for at least six generations.

\section{Triglyceride and glycogen measurements}

All experiments were carried out in controlled growth conditions as described here, unless mentioned otherwise. Fifty $1^{\text {st }}$ instar larvae were collected in fresh food vials avoiding overcrowding within 2-3 $\mathrm{h}$ of hatching. GFP balancers were used wherever required to aid in 
genotyping. Freshly emerged adult male flies were collected (15 per vial) and 5-d-old flies were used for triglyceride and glycogen measurement unless mentioned otherwise. Five flies in triplicates per genotype were homogenized in 0.05\% Tween-20 using Bullet Blender Storm BBY24M from Next Advance. Each experiment was replicated independently and number of independent biological replicates is mentioned for each experiment in the figure legends. The homogenate was heat-inactivated at $70^{\circ} \mathrm{C}$ for 5 min and then centrifuged at 14,000 rpm for 3 min using Eppendorf 5424 centrifuge (FA-45-24-11 rotor). Serum triglyceride determination kit (Cat. no. TR0100) from Sigma-Aldrich was used to quantify triglyceride levels and protein levels were measured using the Quick Start Bradford 1× Dye Reagent (Cat. no. 500-0205) from Bio-Rad. This was followed by colorimetric estimation using TECAN Infinite M200 pro-multimode plate reader in 96-well format. The absorption maximum of 540 and $595 \mathrm{~nm}$ were used for triglyceride and protein content, respectively. Sample preparation for glycogen measurement was similar to triglycerides, following the manufacturer's protocol (Cat. no. MAK016 from Sigma-Aldrich). The absorbance was measured at 570 $\mathrm{nm}$. For triglyceride and glycogen utilisation assay, 5-d-old males (15 per vial) were transferred to vials containing 1\% agar, were collected at the indicated time points, and homogenized as mentioned above. Each experiment was replicated independently and number of replicates $(n)$ is mentioned for each experiment in the figure legends.

\section{Starvation sensitivity assay}

Fifty $1^{\text {st }}$ instar larvae were collected in fresh food vials avoiding overcrowding within 2-3 h of hatching. GFP balancers were used wherever required to aid in genotyping. Freshly emerged adult male flies were collected (15 per vial). For starvation sensitivity assay, 15 ( $5 \mathrm{~d}$ old) male flies were transferred to vials containing $1 \%$ agar and the number of dead flies was counted every $2 \mathrm{~h}$. Multiple vials were set as technical replicates. These experiments were replicated independently and number of independent biological replicates is mentioned in the figure legends.

\section{Life span assay}

Adult life span assay was estimated with data obtained from three independent biological replicates for each genotype. Fifty $1^{\text {st }}$ instar larvae were collected in fresh food vials and freshly emerged adult male flies were collected ( 15 per vial). Multiple vials of adult male flies were set as technical replicates. These flies were flipped into fresh media every $2 \mathrm{~d}$ and the dead flies and the escapers were scored.

\section{Glucose assay}

Fifty $1^{\text {st }}$ instar larvae were collected in fresh food vials. Larvae at third instar stage (five larvae for every prep) were used to isolate hemolymph using Zymo-Spin IIIC (C1006-250) from Zymo Research. $1 \mu \mathrm{l}$ of hemolymph was diluted to $50 \mu \mathrm{l}$ with autoclaved milli-Q water. $100 \mu$ l of glucose assay reagent (Cat. \#no. AGO20) from SigmaAldrich was added and the reaction was incubated at $37^{\circ} \mathrm{C}$ for 30 min. The reaction was stopped with $100 \mu \mathrm{l}$ of $12 \mathrm{~N} \mathrm{H}_{2} \mathrm{SO}_{4}$. The glucose content was analyzed using colorimetric quantification at $540 \mathrm{~nm}$ using TECAN Infinite M200 pro-multi-mode plate reader in 96-well format. Hemolymph glucose measurements were replicated independently and number of replicates is mentioned in the figure legends.

\section{Larval starvation}

Fifty $1^{\text {st }}$ instar larvae were collected in fresh food vials. Third instar non-crawling larvae of the desired genotypes were kept for starvation on $1 \%$ agar vials for $12 \mathrm{~h}$, after washing them with milli-Q water to make sure that there were no traces of media left behind. After $12 \mathrm{~h}$, the larvae were plunged for the qPCR experiments. The starvation experiments were replicated independently and number of replicates is mentioned in the figure legends.

\section{Feeding assay}

Fifty $1^{\text {st }}$ instar larvae were collected in fresh food vials. Larvae at third instar stage (10 each) or 5-d-old flies (five each) were fed for 3 h or 30 min, respectively, with colored food with Orange $\mathrm{G}$ dye (Cat. no. 1936-15-8) from Sigma-Aldrich. The larvae/flies were homogenized using $0.05 \%$ Tween-20. The homogenate was analyzed colorimetrically at $492 \mathrm{~nm}$ using TECAN Infinite M200 pro-multimode plate reader in the 96-well format. The absorbance of the homogenate was directly proportional to the food intake. The feeding experiments were replicated independently and number of replicates is mentioned in the figure legends.

\section{Quantitative RT-PCR}

Fifty $1^{\text {st }}$ instar larvae were collected in fresh food vials. Third instar wandering larvae or 5-d-old male flies for each genotype were collected and were flash-frozen. These experiments were replicated independently and number of replicates ( $n$ ) is mentioned in the figure legends. Total RNA was isolated with QIAGEN RNeasy Plus Mini Kit (Cat. no. 74134) and was quantified using the Qubit RNA HS Assay Kit (Cat. no. Q32852). An equal amount of RNA from each sample was reverse-transcribed using SuperScript III First-Strand Synthesis System (Cat. no. 18080051) from Thermo Fisher Scientific. Quantitative RT-PCR was performed using Bio-Rad CFX96 with the cDNA template, Power SYBR Green PCR Master Mix (Cat. no. 4368702) from Thermo Fisher Scientific and a primer concentration of $312.5 \mathrm{nM}$. The data were normalised to rp49. The sequences of the primers used are mentioned in Table 1.

\section{Immunohistochemistry}

DILP2 peptide corresponding to the sequence TRQRQGIVERC (amino acids 108-118) was used as an immunogen to raise DILP2 polyclonal antibody in rabbit (Eurogentec). Mouse anti-GFP (Cat. \#632375 from Living Colors) was used. About 10 larvae (third instar wandering) were used to dissect the brains in ice-cold $1 \times$ phosphate-buffered saline PBS (Cat. no. P4417 from Sigma-Aldrich) per genotype for each experiment. The dissections were repeated independently and number of replicates $(n)$ is mentioned in the figure legends. The tissue samples were fixed using 4\% PFA (Cat \#P6148 from 
Table 1. List of primer sequences used in the manuscript.

\begin{tabular}{|c|c|}
\hline $4 e b p$ & $\begin{array}{l}\text { FP: 5'-CACTCCTGGAGGCACCA-3' RP: 5'- } \\
\text { GAGTTCCCCTCAGCAAGCAA-3' }\end{array}$ \\
\hline dilp2 & $\begin{array}{l}\text { FP: 5'-GGCCAGCTCCACAGTGAAGT-3' RP: 5'- } \\
\text { TCGCTGTCGGCACCGGGCAT-3' }\end{array}$ \\
\hline dilp3 & $\begin{array}{l}\text { FP: 5'-AAGCTCTGTGTGTATGGCTT-3' RP: 5'- } \\
\text { AGCACAATATCTCAGCACCT-3' }\end{array}$ \\
\hline dilp5 & $\begin{array}{l}\text { FP: 5'-TCCGCCCAGGCCGCAAACTC-3' RP: 5'- } \\
\text { TAATCGAATAGGCCCAAGGT-3' }\end{array}$ \\
\hline dilp6 & $\begin{array}{l}\text { FP: 5'-CGATGTATTTCCCAACAGTTTCG-3' RP: 5'- } \\
\text { AAATCGGTTACGTTCTGCAAGTC-3' }\end{array}$ \\
\hline edem1 & $\begin{array}{l}\text { FP: 5'-CAATCCGGCACAAGCACTACCATGG-3' RP: 5'- } \\
\text { CCTCGCGTATCTGCTCGAAGTTGCT-3' }\end{array}$ \\
\hline eiger & $\begin{array}{l}\text { FP: 5'-CAGCTGATCCCCCTGGTTTT-3' RP: 5'- } \\
\text { GCCAGATCGTTAGTGCGAGA-3' }\end{array}$ \\
\hline inr & $\begin{array}{l}\text { FP: 5'-CACCCCGCTTCTATACTCCA-3' RP: 5'- } \\
\text { GTTAGGATGGTGGCCTGTTC-3' }\end{array}$ \\
\hline nlaz & $\begin{array}{l}\text { FP: 5'-GGTGAATGCGGCCATCAATC-3' RP: 5'- } \\
\text { AATGGCTGCGTCGGGTAAAA-3' }\end{array}$ \\
\hline rp49 & $\begin{array}{l}\text { FP: 5'-GCTAAGCTGTCGCACAAA-3' RP: 5'- } \\
\text { TCCGGTGGGCAGCATGTG-3' }\end{array}$ \\
\hline tace & $\begin{array}{l}\text { FP: 5'-TGGGACACAATTTTGGAGCA-3' RP: 5'-CCTCCTT } \\
\text { GGTCCTCATTTGG-3' }\end{array}$ \\
\hline totA & $\begin{array}{l}\text { FP: 5'-AATTCTTCAACTGCTCTTATGTGC-3' RP: 5'- } \\
\text { TTTGGAGTCATCGTCCTGGG-3' }\end{array}$ \\
\hline upd2 & $\begin{array}{l}\text { FP: 5'-CGGAACATCACGATGAGCGAAT-3' RP: 5'- } \\
\text { TCGGCAGGAACTTGTACTCG-3' }\end{array}$ \\
\hline
\end{tabular}

Sigma-Aldrich) at room temperature for 20 min. PFA was removed and the tissues were washed with PBT $1 \times$ phosphate-buffered saline $+0.1 \%$ Triton X-100 (Cat. no. 161-0407 from Bio-Rad). Blocking solution (PBT + 0.1\% BSA [Cat. no. A2153 from Sigma-Aldrich]) was added to the tissues and the tissues were incubated at room temperature for 45 min. Primary antibody against DILP2 and GFP were diluted in blocking solution in 1:1,000 and 1:500 dilutions, respectively. The samples were incubated with primary antibody overnight at $4^{\circ} \mathrm{C}$ with constant rotation. Then the tissues were washed extensively with PBT and incubated with secondary antibody at room temperature for $2 \mathrm{~h}$. The secondary antibodies Alexa Fluor 488 Goat Anti-Rabbit Immunoglobulin G (IgG). (Cat. no. A27034) and Alexa Fluor 633 Goat Anti-Mouse IgG (Cat. no. A-21050) were diluted in 1: 500 dilution in blocking solution. After $2 \mathrm{~h}$ the samples were washed extensively and mounted with a drop of SlowFade Gold Antifade Reagent with DAPI (Cat. no. S36939) from Thermo Fisher Scientific. The tissues were imaged using a Leica DM6000B upright microscope and processed using ImageJ software. Corrected total cell fluorescence was calculated using the formula Corrected total cell fluorescence $=$ Integrated Density $-($ Area of selected cell $\times$ Mean fluorescence of background readings).

\section{Ex vivo organ co-culture}

For the ex vivo organ co-culture, larval hemolymph was isolated from 10 (third instar crawling) larvae and was incubated with 10 brains from third instar crawling larvae of the desired genotypes in Shields and Sang medium (Cat. no. S3652 from Sigma-Aldrich) at room temperature for $2 \mathrm{~h}$ with constant shaking. The larval brains were then fixed in 4\% PFA and stained for DILP2 as mentioned above and imaged.

\section{Western blotting}

5-d-old adult flies five each of the desired genotype were homogenized in $40 \mu \mathrm{l}$ RIPA buffer with complete, EDTA-free Protease Inhibitor Cocktail (Cat. no. 4693132001 from Sigma-Aldrich). The homogenates were centrifuged at full speed. The samples were denatured in 2× Laemmli sample buffer (Cat. no. 1610737 from Bio-Rad) at $95^{\circ} \mathrm{C}$ and run on $10 \%$ SDS-PAGE. Immobilon Western Chemiluminescent HRP Substrate (Cat. no.WBKLS0050 from Merck; Millipore) was used for antibody detection after blotting on PVDF membrane (Cat. no. 162-0177 from Bio-Rad). The following primary antibodies were used: anti-Egr 1:50 (generous gift from Konrad Basler) and anti-actin 1:3,000 (Cat. no. 612656 from BD Biosciences). For quantification, the intensity of soluble Eiger protein bands was normalised to the intensity of actin bands using Image software.

\section{Statistical analysis}

All the experiments were carried out in biological replicates as indicated and the error bars represent the SEM. The graphs were plotted using GraphPad Prism8 software. Significance was tested using unpaired $t$ test, Welch test, Mann-Whitney test and KruskalWallis test (followed by Dunn's post hoc test) with * representing $P$-value $<0.05,{ }^{* *} P$-value $<0.01,{ }^{* *} P$-value $<0.001$. The starvation and life span datasets were subjected to log-rank test followed by Cox proportional hazard analysis using $\mathrm{R}$ software to analyze the trends in the survival of flies in both the assays.

\section{Data Availability}

Included as data file sets in the supplementary data section.

\section{Supplementary Information}

Supplementary Information is available at https://doi.org/10.26508/lsa. 202101079.

\section{Acknowledgements}

We thank Nisha N Kannan and Smitha Vishnu for critical comments on this manuscript. We thank Sohela Sarkar for her help with the figures. H Pathak was supported by Indian Institute of Science Education and Research Thiruvananthapuram (IISER TVM). PHD fellowship. J Varghese was supported by Ramanujan Fellowship (SR/S2/RJN-140/2011) from Science and Engineering Research Board, Department of Science and Technology, India. This work was also supported by generous funds from Extra-mural Research grant (EMR/2016/004978) from Science and Engineering Research Board, 
Department of Science and Technology, India, and core funding from IISER TVM, Ministry of Human Resources and Development, India.

\section{Author Contributions}

H Pathak: conceptualization, formal analysis, investigation, methodology, and writing-original draft.

J Varghese: conceptualization, resources, supervision, funding acquisition, investigation, methodology, project administration, and writing-original draft, review, and editing.

\section{Conflict of Interest Statement}

The authors declare that they have no conflict of interest.

\section{References}

Accili D, Drago J, Lee EJ, Johnson MD, Cool MH, Salvatore P, Asico LD, José PA, Taylor SI, Westphal H (1996) Early neonatal death in mice homozygous for a null allele of the insulin receptor gene. Nat Genet 12: 106-109. doi:10.1038/ng0196-106

Agrawal N, Delanoue R, Mauri A, Basco D, Pasco M, Thorens B, Léopold P (2016) The Drosophila TNF eiger is an adipokine that acts on insulinproducing cells to mediate nutrient response. Cell Metab 23: 675-684. doi:10.1016/j.cmet.2016.03.003

Andersen DS, Colombani J, Palmerini V, Chakrabandhu K, Boone E, Röthlisberger M, Toggweiler J, Basler K, Mapelli M, Hueber AO, et al (2015) The Drosophila TNF receptor Grindelwald couples loss of cell polarity and neoplastic growth. Nature 522: 482-486. doi:10.1038/ nature14298

Araki K, Nagata K (2011) Protein folding and quality control in the ER. Cold Spring Harb Perspect Biol 3: a007526. doi:10.1101/cshperspect.a007526

Arsic D, Guerin PM (2008) Nutrient content of diet affects the signaling activity of the insulin/target of rapamycin/p70 S6 kinase pathway in the African malaria mosquito Anopheles gambiae. I Insect Physiol 54: 1226-1235. doi:10.1016/j.jinsphys.2008.06.003

Bai H, Kang P, Tatar M (2012) Drosophila insulin-like peptide-6 (dilp6) expression from fat body extends lifespan and represses secretion of Drosophila insulin-like peptide-2 from the brain. Aging Cell 11: 978-985. doi:10.1111/acel.12000

Böhni R, Riesgo-Escovar J, Oldham S, Brogiolo W, Stocker H, Andruss BF, Beckingham K, Hafen E (1999) Autonomous control of cell and organ size by CHICO, a Drosophila homolog of vertebrate IRS1-4. Cell 97: 865-875. doi:10.1016/s0092-8674(00)80799-0

Bonafè M, Barbieri M, Marchegiani F, Olivieri F, Ragno E, Giampieri C, Mugianesi E, Centurelli M, Franceschi C, Paolisso G (2003) Polymorphic variants of insulin-like growth factor I (IGF-I) receptor and phosphoinositide 3-kinase genes affect IGF-I plasma levels and human longevity: Cues for an evolutionarily conserved mechanism of life span control. J Clin Endocrinol Metab 88: 3299-3304. doi:10.1210/ jc.2002-021810

Britton JS, Lockwood WK, Li L, Cohen SM, Edgar BA (2002) Drosophila's insulin / PI3-kinase pathway coordinates cellular metabolism with nutritional conditions. Dev Cell 2: 239-249. doi:10.1016/s1534-5807(02)00117-x

Brogiolo W, Stocker H, Ikeya T, Rintelen F, Fernandez R, Hafen E (2001) An evolutionarily conserved function of the Drosophila insulin receptor and insulin-like peptides in growth control. Curr Biol 11: 213-221. doi:10.1016/s0960-9822(01)00068-9

Broughton SJ, Piper MD, Ikeya T, Bass TM, Jacobson J, Driege $\mathrm{Y}$, Martinez $\mathrm{P}$, Hafen E, Withers DJ, Leevers SJ, et al (2005) Longer lifespan, altered metabolism, and stress resistance in Drosophila from ablation of cells making insulin-like ligands. Proc Natl Acad Sci U S A 102: 3105-3110. doi:10.1073/pnas.0405775102

Broughton SJ, Slack C, Alic N, Metaxakis A, Bass TM, Driege Y, Partridge L (2010) DILP-producing median neurosecretory cells in the Drosophila brain mediate the response of lifespan to nutrition. Aging Cell 9: 336-346. doi:10.1111/j.1474-9726.2010.00558.x

Chouhan NS, Wolf R, Heisenberg M (2017) Starvation promotes odor/feedingtime associations in flies. Learn Mem 24: 318-321. doi:10.1101/ Im.045039.117

Clancy DJ, Gems D, Harshman LG, Oldham S, Stocker H, Hafen E, Leevers SJ, Partridge L (2001) Extension of life-span by loss of CHICO, a Drosophila insulin receptor substrate protein. Science 292: 104-106. doi:10.1126/ science.1057991

Colombani J, Raisin S, Pantalacci S, Radimerski T, Montagne J, Léopold P (2003) A nutrient sensor mechanism controls Drosophila growth. Cell 114: 739-749. doi:10.1016/s0092-8674(03)00713-x

Delanoue R, Meschi E, Agrawal N, Mauri A, Tsatskis Y, McNeill H, Léopold P (2016) Drosophila insulin release is triggered by adipose Stunted ligand to brain Methuselah receptor. Science 353: 1553-1556. doi:10.1126/science.aaf8430

DiAngelo JR, Birnbaum MJ (2009) Regulation of fat cell mass by insulin in Drosophila melanogaster. Mol Cell Biol 29: 6341-6352. doi:10.1128/ MCB.00675-09

Droujinine IA, Perrimon N (2016) Interorgan communication pathways in physiology: Focus on Drosophila. Annu Rev Genet 50: 539-570. doi:10.1146/annurev-genet-121415-122024

Erion R, Sehgal A (2013) Regulation of insect behavior via the insulinsignaling pathway. Front Physiol 4: 353. doi:10.3389/fphys.2013.00353

Fabrizio P, Pozza F, Pletcher SD, Gendron CM, Longo VD (2001) Regulation of longevity and stress resistance by Sch9 in yeast. Science 292: 288-290. doi:10.1126/science.1059497

Fernandez AM, Torres-Alemán I (2012) The many faces of insulin-like peptide signalling in the brain. Nat Rev Neurosci 13: 225-239. doi:10.1038/ nrn3209

Garami A, Zwartkruis FJ, Nobukuni T, Joaquin M, Roccio M, Stocker H, Kozma SC, Hafen E, Bos JL, Thomas G (2003) Insulin activation of Rheb, a mediator of mTOR/S6K/4E-BP signaling, is inhibited by TSC1 and 2. Mol Cell 11: 1457-1466. doi:10.1016/s1097-2765(03)00220-X

Géminard C, Rulifson EJ, Léopold P (2009) Remote control of insulin secretion by fat cells in Drosophila. Cell Metab 10: 199-207. doi:10.1016/ j.cmet.2009.08.002

Ghosh AC, O'Connor MB (2014) Systemic activin signaling independently regulates sugar homeostasis, cellular metabolism, and $\mathrm{pH}$ balance in Drosophila melanogaster. Proc Natl Acad Sci U S A 111: 5729-5734. doi:10.1073/pnas.1319116111

Giannakou ME, Goss M, Jünger MA, Hafen E, Leevers SJ, Partridge L (2004) Long-lived Drosophila with overexpressed dFOXO in adult fat body. Science 305: 361. doi:10.1126/science.1098219

Giannakou ME, Partridge L (2007) Role of insulin-like signalling in Drosophila lifespan. Trends Biochem Sci 32: 180-188. doi:10.1016/j.tibs.2007.02.007

Grönke S, Clarke DF, Broughton S, Andrews TD, Partridge L (2010) Molecular evolution and functional characterization of Drosophila insulin-like peptides. PLoS Genet 6: e1000857. doi:10.1371/journal.pgen.1000857

Hallier B, Schiemann R, Cordes E, Vitos-Faleato J, Walter S, Heinisch IJ, Malmendal A, Paululat A, Meyer H (2016) Drosophila neprilysins control insulin signaling and food intake via cleavage of regulatory peptides. Elife 5: e19430. doi:10.7554/eLife.19430

Haselton A, Sharmin E, Schrader J, Sah M, Poon P, Fridell YW (2010) Partial ablation of adult Drosophila insulin-producing neurons modulates glucose homeostasis and extends life span without insulin resistance. Cell Cycle 9: 3063-3071. doi:10.4161/cc.9.15.12458 
Hirosumi J, Tuncman G, Chang L, Görgün CZ, Uysal KT, Maeda K, Karin M, Hotamisligil GS (2002) A central role for JNK in obesity and insulin resistance. Nature 420: 333-336. doi:10.1038/nature01137

Holzenberger M, Dupont J, Ducos B, Leneuve P, Géloën A, Even PC, Cervera P, Le Bouc Y (2003) IGF-1 receptor regulates lifespan and resistance to oxidative stress in mice. Nature 421: 182-187. doi:10.1038/nature01298

Hong SH, Lee KS, Kwak SJ, Kim AK, Bai H, Jung MS, Kwon OY, Song WJ, Tatar M, Yu K (2012) Minibrain/Dyrk1a regulates food intake through the sir2FOXO-sNPF/NPY pathway in Drosophila and mammals. PLoS Genet 8: e1002857. doi:10.1371/journal.pgen.1002857

Hull-Thompson J, Muffat J, Sanchez D, Walker DW, Benzer S, Ganfornina MD, Jasper H (2009) Control of metabolic homeostasis by stress signaling is mediated by the lipocalin NLaz. PLoS Genet 5: e1000460. doi:10.1371/ journal.pgen.1000460

Hwangbo DS, Gershman B, Tu M-P, Palmer M, Tatar M (2005) Erratum: Drosophila dFOXO controls lifespan and regulates insulin signalling in brain and fat body. Nature 434: 118. doi:10.1038/nature03446

Ikeya T, Galic M, Belawat P, Nairz K, Hafen E (2002) Nutrient-dependent expression of insulin-like peptides from neuroendocrine cells in the CNS contributes to growth regulation in Drosophila. Curr Biol 12: 1293-1300. doi:10.1016/s0960-9822(02)01043-6

Kahn SE, Hull RL, Utzschneider KM (2006) Mechanisms linking obesity to insulin resistance and type 2 diabetes. Nature 444: 840-846. doi:10.1038/nature05482

Kannan K, Fridell YW (2013) Functional implications of Drosophila insulin-like peptides in metabolism, aging, and dietary restriction. Front Physiol 4: 288. doi:10.3389/fphys.2013.00288

Katic M, Kahn CR (2005) The role of insulin and IGF-1 signaling in longevity. Cell Mol Life Sci 62: 320-343. doi:10.1007/s00018-004-4297-y

Kenyon C, Chang J, Gensch E, Rudner A, Tabtiang R (1993) A C. elegans mutant that lives twice as long as wild type. Nature 366: 461-464. doi:10.1038/ $366461 \mathrm{a} 0$

Kim J, Neufeld TP (2015) Dietary sugar promotes systemic TOR activation in Drosophila through AKH-dependent selective secretion of Dilp3. Nat Commun 6: 6846. doi:10.1038/ncomms7846

Kimura KD, Tissenbaum HA, Liu Y, Ruvkun G (1997) daf-2, an insulin receptorlike gene that regulates longevity and diapause in Caenorhabditis elegans. Science 277: 942-946. doi:10.1126/science.277.5328.942

Koyama T, Mirth CK (2016) Growth-blocking peptides as nutrition-sensitive signals for insulin secretion and body size regulation. PLOS Biol 14: e1002551. doi:10.1371/journal.pbio.1002551

Kroeger H, Chiang WC, Lin JH (2012) Endoplasmic reticulum-associated degradation (ERAD) of misfolded glycoproteins and mutant P23H rhodopsin in photoreceptor cells. Adv Exp Med Biol 723: 559-565. doi:10.1007/978-1-4614-0631-0_71

Liu CY, Kaufman RJ (2003) The unfolded protein response. J Cell Sci 116: 1861-1862. doi:10.1242/jcs.00408

Liu JP, Baker J, Perkins AS, Robertson EJ, Efstratiadis A (1993) Mice carrying null mutations of the genes encoding insulin-like growth factor I (Igf-1) and type 1 IGF receptor (Igf1r). Cell 75: 59-72. doi:10.1016/s0092-8674(05)80084-4

Luo J, Lushchak OV, Goergen P, Williams MJ, Nässel DR (2014) Drosophila insulin-producing cells are differentially modulated by serotonin and octopamine receptors and affect social behavior. PLoS One 9: e99732. doi:10.1371/journal.pone.0099732

Molinari M, Calanca V, Galli C, Lucca P, Paganetti P (2003) Role of EDEM in the release of misfolded glycoproteins from the calnexin cycle. Science 299: 1397-1400. doi:10.1126/science.1079474

Nässel DR (2012) Insulin-producing cells and their regulation in physiology and behavior of Drosophila1. Can J Zool 90: 476-488. doi:10.1139/z2012-009

Oh SW, Mukhopadhyay A, Svrzikapa N, Jiang F, Davis RJ, Tissenbaum HA (2005) JNK regulates lifespan in Caenorhabditis elegans by modulating nuclear translocation of forkhead transcription factor/DAF-16. Proc Natl Acad Sci U S A 102: 4494-4499. doi:10.1073/pnas.0500749102

Oldham S (2011) Obesity and nutrient sensing TOR pathway in flies and vertebrates: Functional conservation of genetic mechanisms. Trends Endocrinol Metab 22: 45-52. doi:10.1016/j.tem.2010.11.002

Oldham S, Montagne J, Radimerski T, Thomas G, Hafen E (2000) Genetic and biochemical characterization of dTOR, the Drosophila homolog of the target of rapamycin. Genes Dev 14: 2689-2694. doi:10.1101/gad.845700

Pasco MY, Léopold P (2012) High sugar-induced insulin resistance in Drosophila relies on the lipocalin neural lazarillo. PLoS One 7: e36583. doi:10.1371/journal.pone.0036583

Puig O, Marr MT, Ruhf ML, Tjian R (2003) Control of cell number by Drosophila FOXO: Downstream and feedback regulation of the insulin receptor pathway. Genes Dev 17: 2006-2020. doi:10.1101/ gad.1098703

Rajan A, Perrimon N (2012) Drosophila cytokine unpaired 2 regulates physiological homeostasis by remotely controlling insulin secretion. Cell 151: 123-137. doi:10.1016/j.cell.2012.08.019

Rajan A, Perrimon N (2013) Drosophila cytokine unpaired 2 regulates physiological homeostasis by remotely controlling insulin secretion. Cell 152: 1197. doi:10.1016/j.cell.2013.02.009

Rulifson EJ, Kim SK, Nusse R (2002) Ablation of insulin-producing neurons in flies: Growth and diabetic phenotypes. Science 296: 1118-1120. doi:10.1126/science.1070058

Sano H, Nakamura A, Texada MJ, Truman JW, Ishimoto H, Kamikouchi A, Nibu Y, Kume K, Ida T, Kojima M (2015) Correction: The nutrient-responsive hormone CCHamide-2 controls growth by regulating insulin-like peptides in the brain of Drosophila melanogaster. PLoS Genet 11: e1005481. doi:10.1371/journal.pgen.1005481

Saucedo LJ, Gao X, Chiarelli DA, Li L, Pan D, Edgar BA (2003) Rheb promotes cell growth as a component of the insulin/TOR signalling network. Nat Cell Biol 5: 566-571. doi:10.1038/ncb996

Shimokawa I, Higami Y, Tsuchiya T, Otani H, Komatsu T, Chiba T, Yamaza H (2003) Life span extension by reduction of the growth hormoneinsulin-like growth factor-1 axis: Relation to caloric restriction. FASEB 17: 1108-1109. doi:10.1096/fj.02-0819fje

Shingleton AW, Das J, Vinicius L, Stern DL (2005) The temporal requirements for insulin signaling during development in Drosophila. PLoS Biol 3 : e289. doi:10.1371/journal.pbio.0030289

Slaidina M, Delanoue R, Gronke S, Partridge L, Léopold P (2009) A Drosophila insulin-like peptide promotes growth during nonfeeding states. Dev Cell 17: 874-884. doi:10.1016/j.devcel.2009.10.009

Söderberg JA, Carlsson MA, Nässel DR (2012) Insulin-producing cells in the Drosophila brain also express satiety-inducing cholecystokinin-like peptide, drosulfakinin. Front Endocrinol (Lausanne) 3: 109. doi:10.3389/fendo.2012.00109

Sonntag WE, Carter CS, Ikeno Y, Ekenstedt K, Carlson CS, Loeser RF, Chakrabarty S, Lee S, Bennett C, Ingram R, et al (2005) Adult-onset growth hormone and insulin-like growth factor I deficiency reduces neoplastic disease, modifies age-related pathology, and increases life span. Endocrinology 146: 2920-2932. doi:10.1210/ en.2005-0058

Sudhakar SR, Pathak H, Rehman N, Fernandes J, Vishnu S, Varghese J (2019) Insulin signalling elicits hunger-induced feeding in Drosophila. Dev Biol 459: 87-99. doi:10.1016/j.ydbio.2019.11.013

Sun J, Liu C, Bai X, Li X, Li J, Zhang Z, Zhang Y, Guo J, Li Y (2017) Drosophila FIT is a protein-specific satiety hormone essential for feeding control. Nat Commun 8: 14161. doi:10.1038/ncomms14161

Tatar M, Kopelman A, Epstein D, Tu MP, Yin CM, Garofalo RS (2001) A mutant Drosophila insulin receptor homolog that extends life-span and impairs neuroendocrine function. Science 292: 107-110. doi:10.1126/ science.1057987 
Teleman AA, Maitra S, Cohen SM (2006) Drosophila lacking microRNA miR-278 are defective in energy homeostasis. Genes Dev 20: 417-422. doi:10.1101/gad.374406

Tettweiler G, Miron M, Jenkins M, Sonenberg N, Lasko PF (2005) Starvation and oxidative stress resistance in Drosophila are mediated through the elF4E-binding protein, d4E-BP. Genes Dev 19: 1840-1843. doi:10.1101/ gad. 1311805

Varghese J, Lim SF, Cohen SM (2010) Drosophila miR-14 regulates insulin production and metabolism through its target, sugarbabe. Genes Dev 24: 2748-2753. doi:10.1101/gad.1995910

Wang MC, Bohmann D, Jasper H (2005) JNK extends life span and limits growth by antagonizing cellular and organism-wide responses to insulin signaling. Cell 121: 115-125. doi:10.1016/j.cell.2005.02.030
Wu Q, Zhang Y, Xu J, Shen P (2005) Regulation of hunger-driven behaviors by neural ribosomal 56 kinase in Drosophila. Proc Natl Acad Sci U S A 102 13289-13294. doi:10.1073/pnas.0501914102

Zhang T, Branch A, Shen P (2013) Octopamine-mediated circuit mechanism underlying controlled appetite for palatable food in Drosophila. Proc Natl Acad Sci U S A 110: 15431-15436. doi:10.1073/ pnas.1308816110

(c)

License: This article is available under a Creative Commons License (Attribution 4.0 International, as described at https://creativecommons.org/ licenses/by/4.0/). 